McCarthy et al, 2021

\title{
Birth and delineation of the intestinal stem cell niche
}

Neil McCarthy ${ }^{1,2}$, Guodong Tie ${ }^{1}$, Shariq Madha ${ }^{1}$, Adrianna Maglieri ${ }^{1}$, Judith Kraiczy ${ }^{1,2}$, Ramesh A. Shivdasani ${ }^{1,2,3}$

${ }^{1}$ Department of Medical Oncology and Center for Functional Cancer Epigenetics, Dana-Farber Cancer Institute, Boston, MA 02215, USA

${ }^{2}$ Department of Medicine, Harvard Medical School, Boston, MA 02115, USA

${ }^{3}$ Harvard Stem Cell Institute, Cambridge, MA 02139, USA

\begin{abstract}
Wnt and Rspondin (RSPO) signaling triggers proliferation, and bone morphogenetic protein inhibitors (BMPi) impede differentiation, of intestinal stem cells (ISCs). Here we report that the functional ISC niche is a complex, multi-layered mesenchymal structure that includes distinct smooth muscle populations and describe how that niche organizes early in mouse life. Diverse sub-cryptal cells provide redundant supportive factors, with distinct BMPi and the most potent Wnt agonist, RSPO2, restricted to single cell types. Two functionally opposing elements arise in tandem during a critical period of crypt morphogenesis: a prominent shelf of $\mathrm{BMP}^{+}$sub-epithelial myofibroblasts that promote epithelial differentiation and the muscularis mucosae, a specialized muscle layer generated de novo to supplement other RSPO and BMPi sources. In vivo ablation of smooth muscle, while preserving trophocytes, raises crypt BMP activity and potently limits crypt expansion. Thus, distinct and progressively refined mesenchymal components together create the milieu necessary to propagate crypts during rapid organ growth and to sustain ISCs in the adult niche.
\end{abstract}

\section{Introduction}

The small bowel epithelium contains crypt-like invaginations and finger-like villus projections. Adult multipotent $L g r 5+$ intestinal stem cells (ISCs) proliferate at the crypt base ${ }^{1}$ and relinquish stem-cell properties as they depart this region ${ }^{2}$. A proper epithelial census demands a balance between Wnt/RSPO-dependent ISC self-renewal ${ }^{3}$, and Wnt-independent differentiation driven 
by bone morphogenetic proteins (BMPs) ${ }^{4}$. The crypt base is thus a zone of high Wnt/RSPO and low BMP activity, the same milieu necessary to expand ISCs ex vivo ${ }^{5}$. Inherited polyposis syndromes and mouse studies highlight the fine underlying balance: mutations in human BMP signaling genes SMAD4, BMPR1A or GREM1 result in ISC expansion ${ }^{6-8}$ and forced BMPi expression in mice stimulates ectopic crypt formation ${ }^{9-11}$.

Mouse villus morphogenesis has been elegantly deconstructed ${ }^{12}$ and fetal colon cell types were recently catalogued ${ }^{13}$. At birth, however, the mouse small intestine lacks crypts, which arise over the ensuing $\sim 2$ weeks from epithelial cells sequestered in inter-villus ridges ${ }^{14,15}$. Villi continue to form during this period, as crypts progressively lengthen ${ }^{16}$ and sequester ISCs at the bottom, alongside increasing numbers of Paneth cells. Beyond the morphogenesis of new crypts, fission of existing crypts is a dominant mode of epithelial expansion ${ }^{17-19}$; cell replication ${ }^{20}$, Paneth cell numbers ${ }^{21}$, and crypt bifurcation ${ }^{17,19}$ and monoclonality ${ }^{22}$ all increase dramatically in the third week of mouse life. Thus, the $1^{\text {st }}$ to $3^{\text {rd }}$ weeks after birth represent a dynamic period of epithelial and ISC expansion, likely associated with sculpting of adjacent mesenchyme, the principal determinant of high Wnt and low BMP signaling tone near crypt bottoms ${ }^{23,24}$.

The intestinal sub-epithelium, historically regarded as a myofibroblast tissue ${ }^{25}$, has come

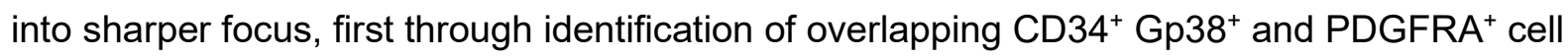
sources of crucial niche factors ${ }^{26-29}$. PDGFRA ${ }^{\text {hi }}$ cells abutting the epithelium resemble telocytes, a diverse cell type with a distinctive morphology found in many organs ${ }^{30,31}$ and proposed as a key source of canonical Wnt ligands ${ }^{32}$. However, these cells express only non-canonical Wnt RNAs $^{13,33}$ and are the same as intestinal sub-epithelial myofibroblasts (ISEMFs) long described in the literature ${ }^{34,35}$; because telocytes lack a defined molecular or functional identity across tissues, here we use the traditional ISEMF nomenclature. Sub-cryptal PDGFRA ${ }^{\text {lo }}$ cells that coexpress the BMPi Grem $1^{10}$ and Rspo3 support ISC expansion in vitro in the absence of exogenous trophic factors, hence earning the name trophocytes ${ }^{33}$. Intestinal smooth muscle (SM) also expresses Grem1 near the crypt base ${ }^{36}$, suggesting that additional cell types might contribute to the functional ISC niche. The totality of mesenchymal cells that perform niche functions or how the niche arises remain unclear.

Here we identify the parallel genesis of murine crypts and the ISC niche. Delineation of this niche between mouse birth and weaning coincides with ISEMF congregation in a dense shelf at the villus base, where the resulting zone of high BMP signaling elicits epithelial differentiation and opposes ISC function. One niche element positioned to counteract that concentrated BMP source is a previously unknown population of trophic factor-expressing cells within the 
superficial circular SM in the muscularis propria. A hallmark postnatal event is the birth of the muscularis mucosae (MM) from resident cells that express platelet-derived growth factor receptor alpha (PDGFRA). Although adult MM is contiguous with perpendicular fibers in the lamina propria and this SM network is considered homogeneous ${ }^{25,35}$, we show that sub-cryptal MM is molecularly distinct, characterized by high levels of Rspo3 and the BMPi Grem2. The MM delimits a distinct sub-cryptal compartment for trophocytes, which alone provide the most potent RSPO ligand, RSPO2, and it complements trophocyte support for ISCs. The MM arises just as crypt fission accelerates, and SM ablation during this period raises crypt BMP activity and markedly attenuates crypt bifurcation. Thus, step-wise development of the niche entails distinct stromal cell types with overlapping expression of trophic factors taking their place at defined distances from the crypt bottom to support lifelong ISC self-renewal.

\section{RESULTS}

Formation and activity of the isthmus ISEMF shelf, a potent BMP source

BMP signaling induces intestinal epithelial maturation ${ }^{4,37}$ and its restriction to adult mouse villi is reflected in absence of phospho-SMAD1/5 (pSMAD) in crypt cells ${ }^{11}$ (Fig. 1a, note that signals are generally stronger in stroma than in the epithelium). We found that epithelial pSMAD staining at embryonic day (E) 16 and postnatal days (P) 1 and 5 is confined to the top half of emerging and established villi (Fig. 1a and Supplemental Data Fig. 1a). We first detected wholevillus epithelial pSMAD at P10 and consistently so by P14 (Fig. 1a). Because this progressive extension of BMP signaling toward the villus base could reflect increased ligand exposure or increased epithelial responsiveness, we tracked the appearance of ISEMFs, which in adults are the dominant source of BMP transcripts ${ }^{13,33}$ and concentrate at villus tips and bases ${ }^{33,38}$. Fetal PDGFRA $^{\text {hi }}$ precursors to this mesenchymal cell population constitute the leading edges of new villi $^{12,39}$ and clusters of GFPhi cells in Pdgfra ${ }^{\text {H2B-Egfp }}$ mice $^{40}$ continue to ensheath villi after birth (Fig. 1b and Supplemental Data Fig. 1b). Through at least the first week of life, however, high ISEMF density was evident only at villus tips; aggregation at the villus base became discernible in some villi at P10, clear by P14, and fully prevalent by P21 (Fig. 1b).

To test the putative role of adult ISEMFs, inferred from mRNA profiles ${ }^{13,33}$, as a functional BMP source, we first cultured adult mouse duodenal crypts in media containing recombinant $(r)$ epidermal growth factor, rRSPO1, and the BMPi rNOG. After organoids had established, we replaced rNOG with various factors or stromal cell types isolated by flow cytometry; without 
exogenous cells or in the presence of GFP- cells from Pdgfra ${ }^{H 2 B-E g f p}$ mice, organoids enlarged into budding structures that house ISCs ${ }^{5}$. Exposure to rBMPs caused their collapse or death, and GFP $^{+}$ISEMFs isolated from Pdgfra ${ }^{\text {H2B-Egfp }}$ mice similarly arrested crypt growth and budding (Fig. 1c and Supplemental Data Fig. 1c-d). Addition of rNOG or rGREM1 to ISEMF-crypt cocultures restored typical budding morphology (Fig. 1c). 5-ethynyl-2'-deoxyuridine (EdU) staining was abundant in the buds of organoids co-cultured with GFP- cells and nearly absent in organoids exposed to ISEMFs or rBMPs (Fig. 1C), which also lost expression of ISC markers Lgr5 and Olfm4 and showed elevated levels of BMP target genes and differentiation markers (Fig. 1d). Thus, ISEMFs impair crypt self-renewal, drive differentiation, and concentrate at the villus base (isthmus) in the $2^{\text {nd }}$ and $3^{\text {rd }}$ weeks of mouse life, coincident with adjacent BMP signaling (Fig. 1e).

\section{Delineation of a multi-component sub-cryptal signaling center}

Neonatal mouse ISCs reside in inter-villus troughs ${ }^{15,41}$ that generate crypts over the next $\sim 2$ weeks $^{16}$, followed by crypt elongation and extensive crypt fission ${ }^{17,19}$. Phalloidin staining of whole-mount intestines confirmed a pre-weaning burst in crypt branching (Fig. 2a), implying that ISC sequestration and function require pre-existing BMPi sources or ones that develop in parallel. Although PDGFRA ${ }^{\circ} \mathrm{CD} 34^{+} \mathrm{Gp} 38^{+}$trophocytes are the principal known source in adult mice $26,27,29,33$, other cells may also contribute. To investigate this possibility, we digested young mouse intestines to favor extraction of cells other than ISEMFs and to capture SM, which has not been examined critically for niche functions. We combined unfractionated wild-type mesenchyme and $\mathrm{GFP}^{+}$cells from Pdgfra ${ }^{\text {H2B-Egfp }}$ mice between P1 and P14 to collect $>61,000$ single cells (Supplemental Data Fig. 2a). After excluding proliferating cells, Cd45/Ptprc ${ }^{+}$ leukocytes, and Peyer's patch-associated follicular cells ${ }^{42}$, the remaining $>51,000$ informative cells included all known mesenchymal populations (Fig. 2b); their sources and distributions from P1 to P14 are shown in Supplemental data Fig. 2b.

High combined Acta2 and Myh11 expression marked pericytes and SM, with striking and unexpected heterogeneity within the latter cells. The scRNA profiles of external longitudinal and circular SM, which constitute the muscularis propria (MP), were dominated by contractility genes and differed little from one another, but a related and distinct subpopulation uniquely expresses two BMPi: Nog and Chrdl1 (Fig. 2c-d). In situ hybridization localized these Pcp4- $\mathrm{Nog}^{+} \mathrm{Chrdl1^{+ }}$ cells to the inner MP surface, distinct from outer Pcp $4^{+} \mathrm{Osr1} 1^{-}$circular and $\mathrm{Pcp} 4^{+} \mathrm{Osr} 1^{+}$ longitudinal SM (Fig. 2e). Noggin, a BMPi that supports ISCs in gain-of-function studies ${ }^{5,11}$, was 
previously mapped to developing fetal SM fibers ${ }^{43}$; we now identify a distinct $\mathrm{Nog}^{+} \mathrm{Chrdl}^{+}$ subpopulation within the MP that, incidentally, expresses less Acta2 and Myh11 than other SM cells (Fig. 2c-f).

BMPi expression also distinguished other SM populations, one of which lacks BMPi while the other expresses high Grem2 (Fig. 2f). In situ hybridization identified these, respectively, as $\mathrm{Hhip}^{+}$lamina propria myocytes (LPM) and $\mathrm{Hhip}^{+} \mathrm{Grem}^{+}$muscularis mucosae (MM) (Fig. $2 \mathrm{~g}-$ note also that P14 and adult LPM are distinct from ISEMFs, Supplemental Data Fig. 2c). Thus, although LPM and MM form a contiguous network of cells presumed to be identical ${ }^{25,35}$, the two cell types display key differences in their potential to counteract BMPs. Together, these findings reveal previously unappreciated molecular SM heterogeneity and the concentration of ISCpromoting factors to the sub-cryptal compartment (Fig. 2h).

Our postnatal mesenchymal survey identified few Wnts, with Wnt4, Wnt5a, and secreted Wnt antagonists present in multiple cell populations, while BMPs are enriched in ISEMFs (Supplemental Data Fig. 2d), similar to adults ${ }^{33}$. Notably, every SM subpopulation expresses at least two BMPi and one RSPO (Fig. 2f) and the most common signature, Grem1+Rspo3, is as

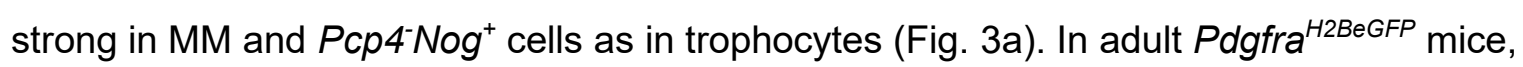
ACTA2 immunostaining revealed MM as a membranous structure distinct from the MP (Fig. 3b). While Grem2 is confined to the MM, Grem1 is expressed in adult MM, trophocytes, and superficial $\mathrm{Nog}^{+} \mathrm{Chrdl1}{ }^{+} \mathrm{MP}$, closely matching the domain of Rspo3 (Fig. 3b). Grem2 $2^{+} \mathrm{MM}$ represents a BMPi source immediately beneath Olfm $4^{+}$ISCs at the crypt base (Fig. $3 \mathrm{c}$ ) while Pdgfra ${ }^{\circ} \mathrm{Grem}^{+}$trophocytes occupy the next sub-cryptal layer between two Grem $1^{+} \mathrm{SM}$ populations: MM and superficial MP (Fig. 3b). To better distinguish these closely apposed cell

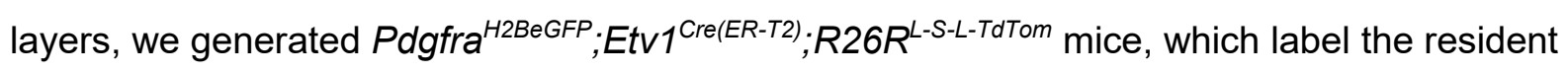
network of axonal projections from ETV1 ${ }^{+}$interstitial cells of Cajal (ICC) ${ }^{44}$ (note: intra-muscular PDGFRA $^{\text {hi }}$ cells are Purkinje neurons ${ }^{45}$ ). PDGFRA ${ }^{\text {lo }}$ trophocytes were sandwiched between the MM and axonal projections into the superficial MP (Supplemental Data Fig. 3a).

Importantly, rNOG and rGREM2 are at least as potent as rGREM1 in supporting ex vivo crypt cultures (Fig. 3d and Supplemental Data Fib. 3b). Our temporal survey also showed increased expression of key signaling factors (Fig. $3 \mathrm{e}-$ including Chrdl1 in superficial $\mathrm{Nog}^{+} \mathrm{MP}$ ) and tissue remodeling genes (Supplemental Data Fig. 3c) from P1 or P2 to P14. Together, these findings point to the sub-cryptal mesenchyme as a layered signaling hub that matures in concert with organ and crypt expansion and where each cellular component co-expresses at least one BMPi and one RSPO gene. 
McCarthy et al, 2021

\section{Postnatal de novo MM genesis}

Three of the four SM populations discriminated by scRNA-seq were present at birth, with little fluctuation in proportions between P1 and P14 (Supplemental Data Fig. 3d); accordingly, BMPi and RSPO elevations in these cells over time reflect increased mRNA per cell. In contrast, MM contribution to the global scRNA dataset was sparse at birth, an observation we corroborated by examining ACTA2 immunostaining over time. Whereas both LPM and MP were present at P1, MM was absent; it appeared sporadically from P5 to P10 and was uniformly evident only by P14 (Fig. 4a-b and Supplemental Data Fig. 3e-f). Curiously, although all adult SM populations lack Pdgfra (and, by extension, GFP in Pdgfra ${ }^{\text {H2B-eGFP intestines }}{ }^{33}$ ), most MM and occasional LPM cells carried GFP ${ }^{+}$nuclei at P14 (Fig. 4b). This suggested that MM and LPM might derive from resident PDGFRA ${ }^{+}$precursors and retain $\mathrm{H} 2 \mathrm{BeGFP}$ (which is stable ${ }^{46}$ ) for some time thereafter.

To test this hypothesis, first we extracted the P14 fraction of $\mathrm{GFP}^{+}$cells from Pdgfra ${ }^{\mathrm{H} 2 \mathrm{~B}-\mathrm{Egfp}}$ mice in our temporal scRNA survey (Fig. 2b). These 8,030 cells included $P d g f r a{ }^{\prime \circ} \mathrm{Cd}^{-1^{-}}$stromal cells, trophocytes, Pdgfra ${ }^{\text {hi }}$ Purkinje neurons ${ }^{45}$ and few ISEMFs, Pdgfra ${ }^{+}$mesothelial and follicle reticular cells (FRCs) ${ }^{42}$ (Fig. 4c and Supplemental Data Fig. 4a), as expected, as well as two distinct pools of Pdgfra $^{+}$Acta2 ${ }^{+} \mathrm{Myh}_{11^{+}}$cells. The smaller population corresponds to Hhip ${ }^{+}$LPM and the larger population matches MM in co-expressing Grem2, Grem1, and Rspo3 (Fig. 4c and Supplemental Data Fig. 4a). Second, we crossed Pdgfra ${ }^{\text {Cre(ER-T2) }}$ mice $^{47}$ with the R26R ${ }^{\text {L-S-L-TdTom }}$ reporter strain ${ }^{48}$, induced TdTom at $\mathrm{P} 1$, and followed the fluorescent label into the derivatives of Pdgfra-expressing cells. Both PDGFRA ${ }^{\text {hi }}$ ISEMFs and PDGFRA ${ }^{\text {lo }}$ stroma were labeled at P10, as expected, and LPM and MM cells also expressed TdTom (Fig. 4d), confirming SM origins in PDGFRA $^{\text {hi }}$ ISEMFs or PDGFRA ${ }^{\text {lo }}$ stroma. ${ }^{49}$ In summary, LPM -which lack RSPOs or BMPi and lie far from crypts- are present at birth, whereas sub-cryptal MM arises postnatally, starting at $\sim$ P5; both populations continue to expand until at least P14.

\section{Complementary MM and trophocyte activities}

The relatively late appearance of $\mathrm{MM}$, coupled with its high expression of trophic factors and proximity to the crypt base, suggests a particular role in supporting ISCs, despite its diminutive dimensions in adult small intestine compared to adult colon (Fig. 5a). To characterize adult MM

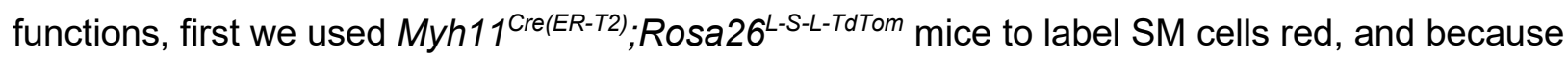
ISEMFs also express Myh1113,33, we crossed in Pdgfra ${ }^{\text {H2B-Egfp }}$ to mark and exclude PDGFRA ${ }^{\text {hi }}$ 
(GFPhi) cells (Fig. 5a - note that, unlike younger animals, the MM in Pdgfra ${ }^{\text {H2BeGFP }}$ adults lacks GFP). After manually stripping the MP, including superficial $\mathrm{Nog}^{+} \mathrm{Chrdl}{ }^{+}$cells, scRNA analysis of adult TdTom ${ }^{+}$GFP- $^{-}$cells identified pericytes, endothelial cells, and abundant myocytes; most myocytes represented Hhip ${ }^{+}$LPM (Supplemental Data Fig. 4b). The small Grem2 $2^{+}$MM population, integrated with previous data from adult mesenchyme ${ }^{33}$, revealed similar distributions of BMPi and Rspo transcripts in MM and trophocytes, with negligible expression in Cd81'Pdgfra ${ }^{\circ}$ cells or LPM (Supplemental Data Fig. 4c). Thus, adult and young sub-cryptal SM show features consistent with their contributions to an ISC signaling center. However, poor MM cell recovery by flow cytometry, coupled with absence of specific surface markers for $\mathrm{Nog}^{+} \mathrm{Chrdl1}{ }^{+} \mathrm{SM}$ cells, precluded their co-culture with crypts to demonstrate niche functions.

We therefore sought to isolate the MM as a membranous tissue, which was possible only in the colon, where MM is thicker than in the small intestine (Fig. 5a). We first used flow cytometry to isolate colonic TdTom ${ }^{+}$GFP- cells, which included a discernible fraction of Grem $1^{+}$Grem $2^{+}$Rspo $^{+}$cells (Fig. 5b). In situ hybridization indicated that this population corresponds to colonic MM; superficial colonic MP expresses Grem1 and also Grem2 (Fig. 5c). Small intestine crypts cultured in Matrigel together with colonic MM peeled away from the submucosa generated spheroids within 1 day, even without any recombinant factors (Supplemental Data Fig. 5a). By the $4^{\text {th }}$ day, spheroids positioned far from the tissue explant were no longer viable, while those positioned close to it had uniformly enlarged (Fig. $5 \mathrm{~d}$ ). Although explanted MM thus clearly supported crypt expansion, the same material from Pdgfra ${ }^{\text {H2B-Egfp }}$ mice showed residual $\mathrm{GFP}^{+}$trophocytes along the undersurface and this unavoidable contamination made it hard to resolve whether MM, trophocytes, or both cells provided the crucial activity.

These findings raised the question whether trophocytes, which alone support robust ISC expansion in vitro ${ }^{33}$, serve a distinct function not redundant with adjacent SM cells. Rspo4 is absent from any cell type, serosal cells express Rspo1 (Supplemental Data Fig. 5b), and several cell types express Rspo3, but only trophocytes express Rspo2 (Fig. 2f), with higher levels at P14 than in neonates (Fig. 3e); in situ hybridization verified this exclusive expression domain (Fig. 5e). RSPO3 is superior to RSPO1 in sustaining organoids ${ }^{27}$, but this trophocyteexclusive family member has not been examined. We found that mouse small intestine crypts required $10 \mathrm{ng} / \mathrm{mL}$ murine $\mathrm{rRSPO} 3$ to generate organoids at top efficiency and $2.5 \mathrm{ng} / \mathrm{mL}$ was ineffective; in contrast, rRSPO2 achieved near-peak efficiency at concentrations as low as 2.5 $\mathrm{ng} / \mathrm{mL}$ and $1 \mathrm{ng} / \mathrm{mL}$ (Fig. 5f). Human RSPO2 also was more potent than RSPO1 or RSPO3 
(Supplemental Data Fig. 5c). Thus, beyond trophocyte expression of Grem1, which is essential for their ex vivo support of organoids ${ }^{33}$, these cells contribute the most potent RSPO.

\section{SM requirement in vivo}

To distinguish SM contributions while overcoming limitations of isolating cells for co-culture, we generated Myh11 ${ }^{\text {Cre-ER(T2) }}$;Rosa26 $6^{\text {L-S-L-DTA }}$ (Myh11;DTA) mice to ablate SM cells in vivo . Although we were especially interested in the role of MM because it arises last, Myh11 Cre-ER(T2) does not target that compartment selectively. It does, however, spare Myh11-Pdgfra ${ }^{\prime o}$ trophocytes and, to monitor those cells, we crossed in Pdgfra ${ }^{H 2 B E G F P}$. We treated pups with tamoxifen at P14, by when the MM has formed, and ACTA2 immunostaining of intestines at P21 (the time we could humanely keep these pups) revealed substantial MM depletion and modest MP atrophy; as expected, ISEMFs and Pdgfralo stroma were preserved (Fig. 6a). We detected no overt epithelial defects, and crypt cell proliferation and expression of the ISC marker Olfm4 were intact (Fig. 6a), likely reflecting redundancies within the signaling center. However, wholemount phalloidin staining in one Myh11;DTA mouse found in fair health at P21 showed fewer crypts and drastically reduced crypt bifurcation compared to Cre- controls (Supplemental Data Fig. 5d). Crypt fission is normally more frequent at $P 16^{17,19}$. At this age, when mice were in good health 2 days after tamoxifen exposure, crypt bifurcation was consistently and substantially reduced (Fig. 6b). pSMAD immunostaining, normally absent from crypts (Fig. 1a), was proportionally and significantly increased (Fig. 6c-d). Crypt fission is one basis for intestinal lengthening and, in keeping with markedly reduced crypt bifurcation, Myh11 ${ }^{\text {Cre-ER(T2) }}$ mouse intestines were shorter than those in Cre- controls (Supplemental Data Fig. 5e). Thus, SM cells -likely including MM and $\mathrm{Nog}^{+} \mathrm{Chrdl}^{+} \mathrm{MP}$ cells- are required to enable epithelial expansion during a critical period of intestinal growth (Fig. 6e).

\section{DISCUSSION}

Mouse intestinal crypts form after birth. Immature ISC precursors located in flat inter-villus epithelium $^{15,41}$ first generate shallow troughs, which deepen into crypts ${ }^{16,20,22}$ that later undergo extensive fission to expand crypt and epithelial mass. Classical ${ }^{17,19-21}$ and recent ${ }^{16}$ studies highlight the P8-P15 interval as a watershed, when crypts and ISCs acquire adult form and cell composition. Our study integrates mesenchymal scRNA profiles with high-resolution anatomic clarity and in vitro and in vivo functional assessment of the emerging ISC niche during this watershed period. We find that sub-epithelial niche components develop alongside crypts, with 
diverse stromal cell types positioned at set distances from the crypt base. ISEMF aggregation at the villus isthmus coincides with increased BMP signaling in adjacent epithelial cells and, presumably in part to counter that elevation, with de novo development of the sub-cryptal MM, a previously unappreciated source of key RSPO and BMPi factors. The MM is molecularly distinct from contiguous, morphologically similar LPM that are present at birth; it arises during crypt morphogenesis from resident PDGFRA cells that contribute simultaneously to postnatal LPM expansion. Another previously unknown $A c t a 2^{10} M y h 11^{10}$ population, sandwiched between MM and trophocytes on the epithelial side and circular and longitudinal SM on the serosal side, uniquely expresses the BMPi Nog and Chdl1. As ISEMFs assemble at high density near crypt tops, sub-cryptal RSPO and BMPi tone increases steadily and the MM is generated de novo to support crypt expansion. Thus, RSPO and BMPi sources position successively and strategically near the crypt base, and by weaning age, the mouse ISC niche is a finely layered arrangement of cells that collectively fulfill known epithelial needs.

ISCs depend on both canonical Wnt/RSPO signaling and BMP inhibition ${ }^{3,4}$ and we identify the niche as a concentrated multi-cellular source of partially redundant RSPOs and BMPi. Study of the ISC niche has heretofore centered largely on candidate sources of canonical Wnt ligands, the presumptive dominant stimulus for crypt cell replication. Few stromal cell types express canonical Wnts and ISEMFs, a putative key source ${ }^{32}$, predominantly express non-canonical Wnts $^{13,29,33}$; that question hence remains unsettled. Furthermore, biochemical ${ }^{50}$, functional ${ }^{51}$, and structural studies ${ }^{52-55}$ point to sequential Wnt and RSPO activity within the same signaling pathway, with Wnts priming ISCs to express crucial RSPO receptors. In addition to one or more $\mathrm{BMPi}$, every mouse sub-cryptal niche component expresses at least one Rspo gene and trophocytes' unique expression of the most potent member, Rspo2, may explain their particularly robust ex vivo support of organoids ${ }^{33}$. Focusing on BMPi, we provide developmental and fine spatial context for prior work showing BMPi activity in driving ISC properties ${ }^{9-11,56}$, polarized BMP and BMPi expression in human colonic mucosa ${ }^{28}$, and that BMP signaling restrains both ISC pool size ${ }^{4}$ and crypt fission ${ }^{57}$ independent of Wnt signaling. Crypt fission, which particularly requires BMP opposition ${ }^{57}$, is markedly compromised in the absence of subepithelial SM.

Overlapping expression of trophic factors in different niche cells implies redundant functions.

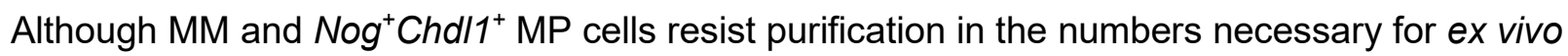
co-cultures, PDGFRA ${ }^{\mathrm{l}} \mathrm{CD} 81^{+}$trophocytes provide necessary BMPi and RSPO activity ex vivo ${ }^{33}$ and tissue containing both MM and trophocytes supported crypt expansion. Trophocytes are 
thus a vital niche component, possibly owing to unique Rspo2 expression, but SM components also contribute and the MM may arise after birth specifically to counteract ISEMF aggregation of at the villus base. Various mouse CRE-driver strains together further help parse individual contributions. Ablation of $\mathrm{Grem} 1^{+}$cells, for example, depletes both trophocytes and SM and affects ISCs severely ${ }^{33}$. Ablation limited to the Myh11 $\mathrm{SM}$ compartment precipitated little overt crypt or ISC dysfunction, but markedly reduced crypt fission. In the future, molecular markers that allow further cell fractionation or precise ablation will complete the growing understanding of individual niche elements.

\section{ACKNOWLEDGMENTS}

Supported in part by National Institutes of Health awards U01DK103152 and R01DK121540 (to R.A.S.) and K01DK125639 (to N.M.). We acknowledge core microscopy and organoid culture services from the Harvard Digestive Disease Center (P30DK034854) and helpful input from E. Manieri and the Dana-Farber Cancer Institute flow cytometry core.

\section{METHODS}

Animals. Pdgfra ${ }^{\text {H2BeGFP }}$ (JAX strain 7669, Ref. ${ }^{40}$ ), Rosa26R ${ }^{\text {LSL-TdTomato }}$ (JAX strain 007909),

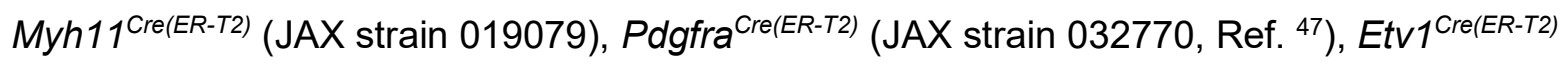
(JAX strain 013048) and Rosa26R $R^{\text {LSL-DTA }(J A X ~ s t r a i n ~ 009669) ~ m o u s e ~ l i n e s ~ w e r e ~ p u r c h a s e d ~ f r o m ~}$ Jackson Laboratories. Mice were considered adults after 8 weeks of age at the time of treatments or cell isolations. All experiments used mice of both sexes and littermates as controls. All animal procedures and experiments were approved and monitored by the Animal Care and Use Committee at the Dana-Farber Cancer Institute.

Mouse treatments. Postnatal Pdgfra ${ }^{\text {Cre(ER-T2) }} ; R 26 R^{\text {LSL-TdTom }}$ and Myh11 ${ }^{\text {Cre(ERT2) }} ;$ Rosa26 $^{\text {LSL-DTA }}$ mice received 1 injection of 4-OH tamoxifen (Sigma-Aldrich, $1 \mathrm{mg}$ per $25 \mathrm{~g}$ body weight) by either gastric gavage (P0) or intra-peritoneal (IP) injection (P14). Myh11 ${ }^{\text {Cre(ER-T2) }}, R 26 R^{L S L-}$

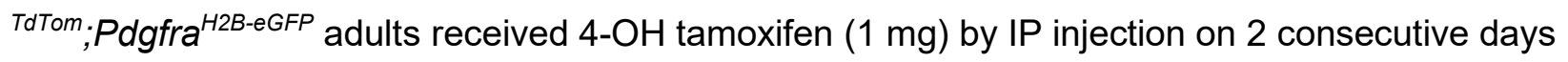
to allow recombination at LoxP sites and were harvested at the indicated times, usually 5 days later. BrDU (10 $\mu \mathrm{g} / \mathrm{gram}$ body weight) was administered by IP injection $1 \mathrm{~h}$ before euthanasia. Postnatal mice were harvested at timepoints indicated in the figure legends. 
Immunohistochemistry and quantitation. Whole-mount tissue immunohistochemistry was performed as described ${ }^{33,57}$. Briefly, proximal small intestines were harvested, pinned onto agarose plates, and fixed overnight in 4\% paraformaldehyde (PFA). In this and all subsequent steps, the tissue was rocked gently. After rinsing in phosphate-buffered saline (PBS), the tissue was placed in $10 \%$, then $20 \%$ sucrose over the course of 1 day, followed by blocking buffer (PBS containing $0.125 \%$ bovine serum albumin, 0.003\% Triton X-100, 0.05\% donkey serum, and $0.0005 \% \mathrm{NaN}_{3}$ ) for $6 \mathrm{~h}$ and overnight in blocking buffer containing 4',6-diamidino-2phenylindole (DAPI). Tissue was rinsed with PBS, cut into 1-mm fragments, placed on glass slides with spacers (Grace Bio-Labs, 654002), and cleared using FocusClear (CelExplorer Labs, FC-101) for 30 min, before applying VectaShield mounting medium (Vector Laboratories) and a coverslip. To generate representative and comprehensive anatomic resolution, images were taken of tissues from at least 3 independent animals.

Proximal small intestine epithelial immunochemistry was performed as described ${ }^{16}$. Fresh tissue was incubated in 5 mM EDTA in Hank's Balanced Salt Solution (HBSS) at room temperature, rocked gently for $5 \mathrm{~min}$, and washed briefly in PBS before separating the epithelium gently from underlying mesenchyme. Tissue was fixed in $4 \%$ PFA at $4{ }^{\circ} \mathrm{C}$ overnight, with subsequent PBS washing and incubation with DAPI to stain nuclei and Phalloidin (Invitrogen, A12381) to visualize filamentous actin. Crypt bifurcation was quantified in 3Drendered images of whole-mount phalloidin stained intestines, reported as a fraction of $>100$ intact crypts.

Routine immunohistochemistry was performed on tissues fixed as described above and placed in OCT compound (Tissue-Tek, 4583). $7 \mu \mathrm{m}$ sections were prepared using a Leica CM3050 cryostat. The following antibodies ( $\mathrm{Ab}$, all at 1:1000 dilution unless indicated) were used: Laminin (Sigma, L9393); GFP (Abcam, ab6662); CD31 (BD Biosciences, 557355); bromo-deoxyuridine (BrDU, Sigma, B5002); Lysozyme (Dako, A0099); Alpha-smooth muscle actin (ACTA2; Abcam, ab5694); PDGFRA (R\&D Systems, AF1062, 1:100); and Alexa Fluorconjugated secondary Ab Goat anti-rat, Goat anti-rabbit, and Donkey anti-Goat IgG (Invitrogen, A11081, A21071, A11058). Signal was amplified using biotin/streptavidin HRP-conjugated secondary Ab (Jackson Immuno, 111-065-003 and 016-030-084) and signal detected using Tyramide Signal Amplification Plus kit (Akoya Biosciences, NEL744001KT, 1:100). Images were taken using a Leica SP5X laser scanning confocal microscope and further processed using Image J Fiji software ${ }^{58}$. 
pSMAD1/5 was immunostained as described ${ }^{59}$. Briefly, antigens were retrieved by boiling slides in citrate buffer for $1 \mathrm{~min}$ and pSMAD1/5 Ab (Cell Signaling, 41D10) was added overnight at $4^{\circ} \mathrm{C}$. pSMAD1/5+ crypts were quantified on images acquired and processed as above. Crypt compartments were delineated with respect to LAM Ab-stained basement membrane and those containing $\geq 1$ pSMAD1/5+ cell were reported as a fraction of total (>80/sample) crypts counted.

In situ RNA hybridization. mRNAs were localized in specific cells by the RNAscope (Advanced Cell Diagnostics) method $^{60}$ on intestines collected from at least 3 different animals. Advanced Cell Diagnostics designed probe sets for Chrdl1, Grem1, Grem2, Hhip, Noggin, Olfm4, Osr1, Pcp4, Rspo1, Rspo2, and Rspo3. After hybridization according to manufacturer's protocols, tissue sections were stained with Laminin and/or GFP Ab. Slides were washed for 5 min in PBS containing $0.1 \%$ Tween-20, blocked for $1 \mathrm{~h}$ at room temperature in PBS containing $5 \%$ normal goat serum, and exposed overnight at $4^{\circ} \mathrm{C}$ to GFP Ab (Abcam, ab6556, 1:100). After multiple 5-min washes in PBS and 90-min incubation with AlexaFluor-conjugated secondary Ab as above (Invitrogen) at room temperature, DAPI was applied and slides were mounted according to the RNAscope protocol. Images were taken using a Leica SP5X laser scanning confocal microscope and processed using ImageJ Fiji software ${ }^{58}$.

Schematic illustrations were generated with BioRender.

Mesenchymal cell isolation and flow cytometry. Mesenchymal cells were isolated from the pooled proximal halves of the small intestine from 3-6 wildtype or Pdgfra ${ }^{\text {H2BeGFP }}$ pups. Whole adult Myh11 ${ }^{\text {Cre(ER-T2) }} ; R 26 R^{\text {L-L-TdTom; }}$ Pdgfra ${ }^{\text {H2B-eGFP }}$ small intestine or colon was used after manual stripping of external muscles (including serosa) and processed as described ${ }^{33}$. The epithelium was denuded by shaking the tissue for $20 \mathrm{~min}$ at $37^{\circ} \mathrm{C}$ in pre-warmed HBSS (Life Technologies) containing $10 \mathrm{mM}$ EDTA. The remaining tissue was rinsed with HBSS, minced using a scalpel, and digested with gentle rocking for $1 \mathrm{~h}$ at $37^{\circ} \mathrm{C}$ in $3 \mathrm{mg} / \mathrm{mL}$ collagenase II (Worthington, LS004176) diluted in HBSS containing 5\% fetal bovine serum (FBS). Extracted cells were centrifuged at $300 \mathrm{~g}$ for $5 \mathrm{~min}$, washed with FACS buffer (PBS containing $0.1 \% \mathrm{BSA}$ ), and leukocytes were depleted in ACK Lysis buffer (Gibco) for 3 min. Washed cells were suspended in FACS buffer, and to deplete epithelial and immune populations, stained with conjugated EPCAM (BioLegends, 118214, 1:100) and CD45 (eBiosciences, 17-0451-82, 1:100) Ab for 20 min at $4^{\circ} \mathrm{C}$. Cells were sorted on a FACSAria IIII flow cytometer, with gating against DAPI (BD 
Pharmingen) to identify live cells. Graphs of isolated cell fractions (Fig. 5b) were generated using FlowJo software v10.

scRNA-seq library preparation, sequencing, alignment, quality control, and data analysis. 5,000 to 10,000 cells isolated by flow cytometry were loaded onto a Chromium Controller (10X Genomics), followed by library preparation according to the manufacturer's recommendations (Single Cell 3' V3 assay) and sequencing on a HiSeq4000 instrument (Illumina). Libraries were de-multiplexed, aligned to the mm10 mouse transcriptome, and unique molecular identifiers (UMIs) were counted using Cell Ranger (10X Genomics) v3.1.1. Data were analyzed using the Seurat package v4.0.3 in R. Cells with $\geq 1,000$ and $\leq 4,000$ detected genes, $>2,500$ and $<15,000$ total transcripts, and $<10 \%$ mitochondrial transcripts were retained. Merged datasets utilized the "merge" function and data were normalized and logtransformed using the "SCTransform" function in Seurat, regressing out mitochondrial read fractions and other confounding variables. Datasets integrated after normalization used the "integrate" function in Seurat. Differential markers were identified in clusters using the "FindAllMarkers" function in Seurat, with parameters: min.pct 0.25 and logfc.threshold 0.25. To identify cell types in each collection, the data were queried for known mesenchymal cell-specific genes $^{33,61}$. All gene expression UMAPs were generated using the "FeaturePlot" function.

For merged datasets (Fig. 2b and Supplemental Data Fig. 2), the "FindNeighbors" function was used and the top 15 principal components were selected. We then identified clusters using the "FindClusters" function in Seurat using a resolution of 2. The "RunUMAP" function was used to reduce the top 15 principal components using the "uwot" method. Based on marker expression, clusters were merged to compile 20 mesenchymal cell populations. Among these, we removed Mki67 $7^{+}$(proliferating), Ptprc $^{+}$(leukocytes), and Ccl19+ (follicle reticular, FRC) cells (Supplemental data Fig. 2) to generate the final merged dataset (Fig. 2b). Correlation heatmaps (Fig. 2d) and gene lists (Supplemental Data Table 1) were generated by separating SM populations, running the "FindAllMarkers" function in Seurat, and plotting the top 10 and top 50 marker genes for each cell type. Differential gene expression (Fig.3e) between P14 and P1/2 was identified within subsets for each cell type using the "FindMarkers" function and dot plots depicting $\log _{2}$ fold-differences were generated using code based on the "DotPlot" function within the ggplot2 package in R. Heatmaps in Supplemental Data Fig. 3b show average cell expression of the top 50 differentially expressed genes at each harvest between P1 or P2 and P14. Integrated analysis (Supplemental Data Fig. 4) included published adult scRNA-seq data ${ }^{33}$ (Gene Expression Omnibus, GSE130681). 
McCarthy et al, 2021

In vitro co-culture conditions, imaging, quantitation, and analysis. Unfractionated mesenchyme extracted from adult $P d g f r a^{H 2 B e G F P}$ mice was plated on non-pyrogenic, gas plasma surface-treated polystyrene tissue culture plates (Falcon) in Dulbecco's Modified Eagle and F12 media (Gibco, 12634-010) supplemented with penicillin, streptomycin, Glutamax, HEPES buffer, and $10 \%$ FBS (Basal media + FBS). The medium was replaced $24 \mathrm{~h}$ after plating; 5 to 7 days later, cells were removed using $0.25 \%$ Trypsin/EDTA (Corning), washed in FACS buffer, and GFPhi $^{\text {hi }}$ (ISEMFs) and GFP- cell fractions were harvested by flow cytometry. For co-cultures with whole colonic MM (Fig. 5), external muscle (including serosa) was carefully removed; MM was stripped using forceps and placed into Matrigel together with small intestine crypts isolated in parallel as described ${ }^{62}$.

For passaged co-cultures (Fig. 1), crypts were plated for 2 days in complete basal media supplemented with N2, B27 and N-acetylcysteine as described ${ }^{62}$, EGF (Thermo Fisher, 50 ng/mL), RSPO1 (293T-HA-Rspol-Fc cell culture supernatant, 10\%), and rNOG (Peprotech, 100 $\mathrm{ng} / \mathrm{mL}$ ), then removed from Matrigel in Cell Recovery Solution (Corning) for $10 \mathrm{~min}$ at $4^{\circ} \mathrm{C}$ and recovered by centrifugation at $200 \mathrm{~g}$ for $15 \mathrm{~min}$. 50-100 crypts were replated in $20 \mu$ Matrigel drops in 24-well tissue culture plates, together with $2 \times 10^{4}$ mesenchymal cells harvested, cultured, and purified as described above. Complete Basal Media was replaced containing EGF, RSPO1 (293T-HA-Rspol-Fc cell culture supernatant, 10\%), and either rNOG, rGREM1 (Thermo Fisher, $100 \mathrm{ng} / \mathrm{ml}$ ), or BMP2 (Peprotech \#120-02, $50 \mathrm{ng} / \mathrm{mL}$ ) and BMP7 (R\&D\#5666-BP, 50 $\mathrm{ng} / \mathrm{mL}$ ). Organoids were imaged using an Olympus CKX53 microscope and fluorescent organoid images were captured with a Nikon Eclipse T/2 microscope. Phenotypes were scored as described (Supplemental data Fig. 1c) from 3 or 4 experiments with 2 or 3 technical replicates (results averaged) per group per experiment. RNA was harvested at $48 \mathrm{~h}$ and qRTPCR was performed on 3 or 4 replicates from each treatment group; mRNA expression values are represented relative to Gapdh $\left(2^{-\mathrm{ACT}}\right)$ and relative to the no-cells group.

For culture experiments with BMPi (Fig. 3d), 100 isolated crypts were plated in Matrigel drops in media containing a sub-optimal concentration of RSPO1 (culture supernatant from 293T-HA-Rspo1-Fc cells, 1\%), EGF (50 ng/mL), and indicated concentrations of rNOG, rGREM1, or rGREM2 (R\&D\#2069-PR). For culture experiments with RSPOs (Fig. 5f), 100 isolated crypts were plated in Matrigel drops in basal condition media containing rNOG (100 $\mathrm{ng} / \mathrm{mL}$ ), EGF (50 ng/mL), and the indicated concentrations of mRSPO2, mRSPO3 (R\&D, 6946RS and 4120-RS), hRSPO1, hRSPO2, or hRSPO3 (R\&D, 4645-RS, 3266-RS, and 3500-RS). 
Organoid structures were counted with a Nikon Eclipse TS100 microscope 5 days after plating. All experiments included 3 technical replicates, with their averages reported as 1 biological replicate and 3 biological replicates are reported per condition. Figure $5 \mathrm{~d}$ and Supplemental Data Fig. 5a were compiled as composite using Microsoft 365 ProPlus Office PowerPoint.

Statistics and reproducibility. Statistical analyses were performed using Prism software package v7.03 (GraphPad). The number of independent replicates, statistical methods, and P values are given in the respective figure legends. No sample size estimations and no blinding were performed.

\section{REFERENCES}

1 Barker, N. et al. Identification of stem cells in small intestine and colon by marker gene Lgr5. Nature 449, 1003-1007 (2007).

2 Ritsma, L. et al. Intestinal crypt homeostasis revealed at single-stem-cell level by in vivo live imaging. Nature 507, 362-365, doi:10.1038/nature12972 (2014).

3 Clevers, $H$. The intestinal crypt, a prototype stem cell compartment. Cell 154, 274-284 (2013).

$4 \quad$ Qi, Z. et al. BMP restricts stemness of intestinal Lgr5(+) stem cells by directly suppressing their signature genes. Nat Commun 8, 13824, doi:10.1038/ncomms13824 (2017).

5 Sato, T. et al. Single Lgr5 stem cells build crypt-villus structures in vitro without a mesenchymal niche. Nature 459, 262-265 (2009).

6 Howe, J. R. et al. Mutations in the SMAD4/DPC4 gene in juvenile polyposis. Science 280, 1086-1088, doi:10.1126/science.280.5366.1086 (1998).

7 Howe, J. R. et al. Germline mutations of the gene encoding bone morphogenetic protein receptor 1A in juvenile polyposis. Nat Genet 28, 184-187, doi:10.1038/88919 (2001).

8 Jaeger, E. et al. Hereditary mixed polyposis syndrome is caused by a $40-\mathrm{kb}$ upstream duplication that leads to increased and ectopic expression of the BMP antagonist GREM1. Nat Genet 44, 699-703 (2012).

9 Batts, L. E., Polk, D. B., Dubois, R. N. \& Kulessa, H. Bmp signaling is required for intestinal growth and morphogenesis. Dev Dyn 235, 1563-1570 (2006).

10 Davis, H. et al. Aberrant epithelial GREM1 expression initiates colonic tumorigenesis from cells outside the stem cell niche. Nat Med 21, 62-70 (2015).

11 Haramis, A. P. et al. De novo crypt formation and juvenile polyposis on BMP inhibition in mouse intestine. Science 303, 1684-1686 (2004).

12 Walton, K. D. et al. Villification in the mouse: BMP signals control intestinal villus patterning. Development 143, 427-436, doi:10.1242/dev.130112 (2016).

13 Brugger, M. D., Valenta, T., Fazilaty, H., Hausmann, G. \& Basler, K. Distinct populations of crypt-associated fibroblasts act as signaling hubs to control colon homeostasis. PLoS Biol 18, e3001032, doi:10.1371/journal.pbio.3001032 (2020).

14 Calvert, R. \& Pothier, P. Migration of fetal intestinal intervillous cells in neonatal mice. Anat Rec 227, 199-206, doi:10.1002/ar.1092270208 (1990).

15 Itzkovitz, S., Blat, I. C., Jacks, T., Clevers, H. \& van Oudenaarden, A. Optimality in the development of intestinal crypts. Cell 148, 608-619, doi:10.1016/j.cell.2011.12.025 (2012). 
16 Sumigray, K. D., Terwilliger, M. \& Lechler, T. Morphogenesis and compartmentalization of the intestinal crypt. Dev Cell 45, 183-197 e185, doi:10.1016/j.devcel.2018.03.024 (2018).

17 Cheng, H. \& Bjerknes, M. Whole population cell kinetics and postnatal development of the mouse intestinal epithelium. Anat Rec 211, 420-426, doi:10.1002/ar.1092110408 (1985).

18 Maskens, A. P. \& Dujardin-Loits, R. M. Kinetics of tissue proliferation in colorectal mucosa during post-natal growth. Cell Tissue Kinet 14, 467-477, doi:10.1111/j.13652184.1981.tb00553.x (1981).

19 St Clair, W. H. \& Osborne, J. W. Crypt fission and crypt number in the small and large bowel of postnatal rats. Cell Tissue Kinet 18, 255-262, doi:10.1111/j.13652184.1985.tb00655.x (1985).

20 Al-Nafussi, A. I. \& Wright, N. A. Cell kinetics in the mouse small intestine during immediate postnatal life. Virchows Arch B Cell Pathol Incl Mol Pathol 40, 51-62, doi:10.1007/BF02932850 (1982).

21 Bry, L. et al. Paneth cell differentiation in the developing intestine of normal and transgenic mice. Proc Natl Acad Sci USA 91, 10335-10339, doi:10.1073/pnas.91.22.10335 (1994).

22 Schmidt, G. H., Winton, D. J. \& Ponder, B. A. Development of the pattern of cell renewal in the crypt-villus unit of chimaeric mouse small intestine. Development 103, 785-790 (1988).

23 Farin, H. F., Van Es, J. H. \& Clevers, H. Redundant sources of Wnt regulate intestinal stem cells and promote formation of Paneth cells. Gastroenterology 143, 1518-1529 (2012).

24 McCarthy, N., Kraiczy, J. \& Shivdasani, R. A. Cellular and molecular architecture of the intestinal stem cell niche. Nat Cell Biol 22, 1033-1041, doi:10.1038/s41556-020-0567-z (2020).

25 Powell, D. W., Pinchuk, I. V., Saada, J. I., Chen, X. \& Mifflin, R. C. Mesenchymal cells of the intestinal lamina propria. Annual review of physiology 73, 213-237, doi:10.1146/annurev.physiol.70.113006.100646 (2011).

26 Stzepourginski, I. et al. CD34+ mesenchymal cells are a major component of the intestinal stem cells niche at homeostasis and after injury. Proc Natl Acad Sci USA 114, E506-E513 (2017).

27 Greicius, G. et al. PDGFRalpha(+) pericryptal stromal cells are the critical source of Wnts and RSPO3 for murine intestinal stem cells in vivo. Proc Natl Acad Sci USA 115, E3173-E3181 (2018).

28 Kosinski, C. et al. Gene expression patterns of human colon tops and basal crypts and BMP antagonists as intestinal stem cell niche factors. Proc Natl Acad Sci USA 104, 15418-15423 (2007).

29 Kim, J. E. et al. Single cell and genetic analyses reveal conserved populations and signaling mechanisms of gastrointestinal stromal niches. Nat Commun 11, 334, doi:10.1038/s41467-019-14058-5 (2020).

30 Cretoiu, D., Cretoiu, S. M., Simionescu, A. A. \& Popescu, L. M. Telocytes, a distinct type of cell among the stromal cells present in the lamina propria of jejunum. Histol Histopathol 27, 1067-1078, doi:10.14670/HH-27.1067 (2012).

31 Popescu, L. M. \& Faussone-Pellegrini, M. S. TELOCYTES - a case of serendipity: the winding way from Interstitial Cells of Cajal (ICC), via Interstitial Cajal-Like Cells (ICLC) to TELOCYTES. J Cell Mol Med 14, 729-740, doi:10.1111/j.1582-4934.2010.01059.x (2010).

32 Shoshkes-Carmel, M. et al. Subepithelial telocytes are an important source of Wnts that supports intestinal crypts. Nature 557, 242-246 (2018). 
33 McCarthy, N. et al. Distinct mesenchymal cell populations generate the essential intestinal BMP signaling gradient. Cell Stem Cell 26, 391-402 e395, doi:10.1016/j.stem.2020.01.008 (2020).

34 Powell, D. W., Adegboyega, P. A., Di Mari, J. F. \& Mifflin, R. C. Epithelial cells and their neighbors I. Role of intestinal myofibroblasts in development, repair, and cancer. $A m \mathrm{~J}$ Physiol Gastrointest Liver Physiol 289, G2-7 (2005).

35 Roulis, M. \& Flavell, R. A. Fibroblasts and myofibroblasts of the intestinal lamina propria in physiology and disease. Differentiation 92, 116-131 (2016).

36 Koppens, M. A. J. et al. Bone Morphogenetic Protein Pathway Antagonism by Grem1 Regulates Epithelial Cell Fate in Intestinal Regeneration. Gastroenterology 161, 239-254 e239, doi:10.1053/j.gastro.2021.03.052 (2021).

37 Chen, L. et al. A reinforcing HNF4-SMAD4 feed-forward module stabilizes enterocyte identity. Nat Genet 51, 777-785 (2019).

38 Bahar Halpern, K. et al. Lgr5+ telocytes are a signaling source at the intestinal villus tip. Nat Commun 11, 1936, doi:10.1038/s41467-020-15714-x (2020).

39 Karlsson, L., Lindahl, P., Heath, J. K. \& Betsholtz, C. Abnormal gastrointestinal development in PDGF-A and PDGFR-(alpha) deficient mice implicates a novel mesenchymal structure with putative instructive properties in villus morphogenesis. Development 127, 3457-3466 (2000).

40 Hamilton, T. G., Klinghoffer, R. A., Corrin, P. D. \& Soriano, P. Evolutionary divergence of platelet-derived growth factor alpha receptor signaling mechanisms. Mol Cell Biol 23, 4013-4025 (2003).

41 Kim, T. H., Escudero, S. \& Shivdasani, R. A. Intact function of Lgr5 receptor-expressing intestinal stem cells in the absence of Paneth cells. Proc Natl Acad Sci USA 109, 39323937, doi:10.1073/pnas.1113890109 (2012).

42 Link, A. et al. Fibroblastic reticular cells in lymph nodes regulate the homeostasis of naive T cells. Nat Immunol 8, 1255-1265, doi:10.1038/ni1513 (2007).

43 Huycke, T. R. et al. Genetic and mechanical regulation of intestinal smooth muscle development. Cell 179, 90-105 e121, doi:10.1016/j.cell.2019.08.041 (2019).

44 Chi, P. et al. ETV1 is a lineage survival factor that cooperates with KIT in gastrointestinal stromal tumours. Nature 467, 849-853, doi:10.1038/nature09409 (2010).

45 Kurahashi, M., Nakano, Y., Hennig, G. W., Ward, S. M. \& Sanders, K. M. Plateletderived growth factor receptor alpha-positive cells in the tunica muscularis of human colon. J Cell Mol Med 16, 1397-1404, doi:10.1111/j.1582-4934.2011.01510.x (2012).

46 Kanda, T., Sullivan, K. F. \& Wahl, G. M. Histone-GFP fusion protein enables sensitive analysis of chromosome dynamics in living mammalian cells. Curr Biol 8, 377-385, doi:10.1016/s0960-9822(98)70156-3 (1998).

47 Chung, M. I., Bujnis, M., Barkauskas, C. E., Kobayashi, Y. \& Hogan, B. L. M. Nichemediated BMP/SMAD signaling regulates lung alveolar stem cell proliferation and differentiation. Development 145, dev163014, doi:10.1242/dev.163014 (2018).

48 Madisen, L. et al. A robust and high-throughput Cre reporting and characterization system for the whole mouse brain. Nat Neurosci 13, 133-140, doi:10.1038/nn.2467 (2010).

49 La Manno, G. et al. RNA velocity of single cells. Nature 560, 494-498, doi:10.1038/s41586-018-0414-6 (2018).

50 Yan, K. S. et al. Non-equivalence of Wnt and R-spondin ligands during Lgr5+ intestinal stem-cell self-renewal. Nature 545, 238-242, doi:10.1038/nature22313 (2017).

51 Storm, E. E. et al. Targeting PTPRK-RSPO3 colon tumours promotes differentiation and loss of stem-cell function. Nature 529, 97-100, doi:10.1038/nature16466 (2016). 
52 Chen, P. H., Chen, X., Lin, Z., Fang, D. \& He, X. The structural basis of R-spondin recognition by LGR5 and RNF43. Genes Dev 27, 1345-1350, doi:10.1101/gad.219915.113 (2013).

53 Peng, W. C. et al. Structure of stem cell growth factor R-spondin 1 in complex with the ectodomain of its receptor LGR5. Cell Rep 3, 1885-1892, doi:10.1016/j.celrep.2013.06.009 (2013).

54 Wang, D. et al. Structural basis for R-spondin recognition by LGR4/5/6 receptors. Genes Dev 27, 1339-1344, doi:10.1101/gad.219360.113 (2013).

55 Zebisch, M. et al. Structural and molecular basis of ZNRF3/RNF43 transmembrane ubiquitin ligase inhibition by the Wnt agonist R-spondin. Nat Commun 4, 2787, doi:10.1038/ncomms3787 (2013).

$56 \mathrm{He}, \mathrm{X}$. C. et al. BMP signaling inhibits intestinal stem cell self-renewal through suppression of Wnt-beta-catenin signaling. Nat Genet 36, 1117-1121 (2004).

57 Auclair, B. A., Benoit, Y. D., Rivard, N., Mishina, Y. \& Perreault, N. Bone morphogenetic protein signaling is essential for terminal differentiation of the intestinal secretory cell lineage. Gastroenterology 133, 887-896 (2007).

58 Bernier-Latmani, J. \& Petrova, T. V. High-resolution 3D analysis of mouse smallintestinal stroma. Nat Protoc 11, 1617-1629, doi:10.1038/nprot.2016.092 (2016).

59 Schindelin, J. et al. Fiji: an open-source platform for biological-image analysis. Nat Methods 9, 676-682, doi:10.1038/nmeth.2019 (2012).

60 Nerurkar, N. L., Mahadevan, L. \& Tabin, C. J. BMP signaling controls buckling forces to modulate looping morphogenesis of the gut. Proc Natl Acad Sci USA 114, 2277-2282, doi:10.1073/pnas.1700307114 (2017).

61 Wang, F. et al. RNAscope: a novel in situ RNA analysis platform for formalin-fixed, paraffin-embedded tissues. J Mol Diagn 14, 22-29, doi:10.1016/j.jmoldx.2011.08.002 (2012). 
McCarthy et al, 2021

\section{FIGURE LEGENDS}

Figure 1: Postnatal epithelial BMP signaling coincides with isthmus ISEMF condensation. a, Representative pSMAD1/5 immunostaining (red, side-by-side greyscale) in mouse proximal small intestines at the indicated postnatal $(P)$ days. Boxed regions are magnified in adjoining greyscale images. Epithelial nuclear pSMAD1/5 (dashed red outlines) indicate progressive pSMAD activity over whole villus units in the first 2 weeks of life, while crypts (dashed white outlines) fail to activate pSMAD1/5. Scale bar $50 \mu \mathrm{m}$.

b, Representative images of $P d g f^{2}{ }^{H 2 B e G F P}$ mouse proximal small intestines at indicated ages. GFPhi $^{\text {hi cells are ISEMFs }}{ }^{33}$ and brackets outline their progressive concentration at the crypt-villus junction (isthmus). Backets b' and b" mark levels of orthogonal views shown to the right, demonstrating low (P5) and high (P14) isthmus ISEMF density over time. Scale bar $50 \mu \mathrm{m}$. c, 2 day-passaged organoids cultured with recombinant (r) RSPO1, EGF, and indicated cell types from Pdgfra ${ }^{\text {H2BeGFP }}$ mouse intestines. Representative organoids are shown $48 \mathrm{~h}$ later (scale bar $100 \mu \mathrm{m}$ ). Co- culture with GFP- or no cells produced budding structures, whereas crypts co-cultured with ISEMFs or rBMPs collapsed and were rescued by rNOG (N) or rGREM1 (G1). Results from 3 or 4 biological replicates are quantified in the right graph. Representative images of EdU-stained organoids are shown below (scale bar $50 \mu \mathrm{m}$ ), demonstrating markedly reduced cell replication in organoids co-cultured with ISEMFs or rBMPs.

d, qRT-PCR analysis of organoids after $48 \mathrm{~h}$ co-culture ( $\mathrm{n}=3$ or 4 biological replicates). BMP- or ISEMF-exposed organoids showed reduced expression of ISC markers and elevated markers of BMP activation and epithelial differentiation. Transcript levels are represented relative to no cells (co-culture). Statistical comparisons use one-way ANOVA followed by Dunnett's posttest at **** $\mathrm{P}<0.0001,{ }^{* * *} \mathrm{P}<0.001,{ }^{* *} \mathrm{P}<0.01$ or ${ }^{*} \mathrm{P}<0.05$.

e, Schema of postnatal increases in epithelial pSMAD1/5 and isthmus ISEMF congregation.

\section{Figure 2: Diverse smooth muscle (SM) populations in postnatal intestinal mesenchyme.}

a, Representative images of whole mount phalloidin-stained epithelium at the indicated ages, showing increasing crypt length (white arrowheads) and bifurcation (fission, red arrowheads). b, Uniform manifold approximation and projection (UMAP) of scRNA libraries from whole mesenchyme and GFP ${ }^{+}$cells from $P$ dgfra ${ }^{\text {H2BeGFP }}$ mice, representing a total of 51,084 cells from P1/P2, P4/P5, P9, and P14. MP, muscularis propria; LPM, lamina propria myocytes; MM, muscularis mucosae; ISEMFs, intestinal subepithelial myofibroblasts; ICC, interstitial cells of Cajal; LECs, lymphatic endothelium; ECs, (vascular) endothelium; NCC, neural crest cells. 
c, Overlay of aggregate Myh11+Acta2 expression (red) on the UMAP plot, showing the SM markers in pericytes, MP, LPM, and MM.

d, Top genes identified as discriminating markers for each indicated SM cell population.

e, RNAscope in situ hybridization with the indicated probes (green dots, red=LAMININ Ab stain), showing 3 distinct MP cell layers (outlined with brackets) that correspond to scRNA-identified subpopulations (dashed green box in c): superficial Nog-and Chrdl1-expressing cells, inner circular SM expressing high Pcp4, and outer longitudinal SM expressing Osr1. Dashed white outlines demarcate crypt epithelium. Scale bar $50 \mu \mathrm{m}$.

f, Average per-cell expression of selected BMPi and Rspo genes across all cell types present in the combined postnatal mesenchymal scRNA-seq analysis shown in Figure $2 b$.

g, Projection of Grem2 (red) and Hhip (blue) expression on the UMAP section that represents MM and LPM (dashed blue box in c), alongside RNAscope in situ hybridization at P14 with Hhip (red) and Grem2 (green) probes. Hhip marks both LPM (arrow) and MM (arrowhead), whereas Grem2 selectively marks MM. Scale bars $50 \mu \mathrm{m}$.

h, Diagram distinguishing LPM from Grem2 ${ }^{+} \mathrm{MM}$ and $\mathrm{Nog}^{+} \mathrm{Chdl1}{ }^{+}$cells from circular Pcp ${ }^{+} \mathrm{SM}$ in P14 mouse small intestines. STable summarizes expression of key trophic factors.

Figure 3: Sub-cryptal mesenchymal cells together constitute an ISC signaling center.

a, Overlay of aggregate Grem1 and Rspo3 expression on the global postnatal UMAP, showing high dual expression in an MP subpopulation, trophocytes (Troph.), and MM.

b, Delineation of mesenchymal cell populations in adult Pdgfra ${ }^{\text {H2BeGFP }}$ duodenum. ACTA2 (smooth muscle) and CD31 (vasculature) Ab stains identify thin MM (arrowheads) and LPM, distinct from the thick MP. In situ hybridization reveals Grem1 (red) in sub-cryptal trophocytes (asterisk, GFP+), MM, and superficial MP; Grem2 (yellow) in MM, superficial MP, and interstitial cells of Cajal (ICC), in line with scRNA data (Fig. 2f); and Rspo3 (red and adjoining greyscale) in MM, MP, and scattered submucosal cells. White or red dashes demarcate crypts and brackets indicate expression domains. Scale bars $50 \mu \mathrm{m}$.

c, Grem2 expression revealed by in situ hybridization (GFP) in adult duodenal MM, directly beneath Olfm4 $4^{+}$(RFP) ISCs (arrowheads). External muscle (MP) was manually removed before staining. The bottom image is the same as the top, with DAPI signal excluded. Scale bar $50 \mu \mathrm{m}$. d, Quantification of total number of small intestine budding organoids generated after 5 days of culture in a sub-optimal concentration of rRSPO1 (see Methods) and $50 \mathrm{ng} / \mathrm{mL}$ or $100 \mathrm{ng} / \mathrm{mL}$ of 
the indicated BMP inhibitors ( $n=3$ biological replicates). One-way ANOVA with Dunnett's test shows significance of all conditions to at least $p<0.01$ compared to controls without BMPi. e, $\log _{2}$ fold increase from P1/2 to P14 in average per-cell mRNA expression of the indicated ISC-trophic genes. Significant increases ( $p<0.05$, circled) show BMPi and Rspo elevation in diverse postnatal sub-cryptal cell types.

Figure 4: Muscularis mucosae (MM) arises postnatally from native Pdgfra+ precursors. a, Representative ACTA2 staining of proximal Pdgfra ${ }^{H 2 B e G F P}$ small intestine at the indicated ages. LPM (arrow) is present by P1, but MM (arrowheads) arises between P5 and P10.

b, GFP ${ }^{+}$nuclei are evident in LPM (arrow) and MM (arrowhead) in Pdgfra ${ }^{\text {H2BeGFP }}$ proximal small intestine at $\mathrm{P} 14$, in agreement with scRNA-seq data on purified $\mathrm{GFP}^{+}$cells.

c, Expression of Acta2, Myh11 (aggregate, red), Grem2 (blue), and Hhip (red) overlaid on the composite UMAP plot. FRC, fibroblastic reticular cells; SM, smooth muscle.

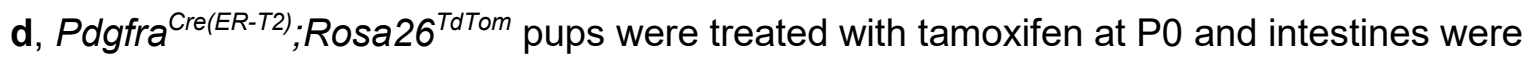
examined at P1 and P10. LPM (arrow) and MM (arrowhead) showed Pdgfra lineage tracing at $\mathrm{P} 10$. White dotted lines outline the epithelial-mesenchymal boundary. Dashed boxes at P1 and $\mathrm{P} 10$ are magnified to their right, in greyscale at $\mathrm{P} 1$. All scale bars $50 \mu \mathrm{m}$.

Figure 5: Adult MM and trophocytes together support organoid growth in vitro.

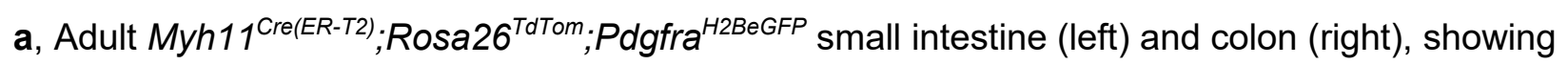
GFP $^{+}$Tom $^{+}$ISEMFs (dashed box in small intestine is detailed below) and Tom ${ }^{+}$SM cells. Small intestine (arrowheads) and colonic (bracket) MM are highlighted. Scale bars (a, b, c, e) $50 \mu \mathrm{m}$. b, FACS plot from isolation of colonic TdTom ${ }^{+}$GFP- mesenchymal cells, followed by scRNA analysis, where the UMAP plot of 3,592 cells shows aggregate Grem1, Grem2, and Rspo3 expression in the small MM population, distinct from non-MM smooth muscle, endothelial cells (ECs) or pericytes.

c, Grem1 (red) and Grem2 (yellow) co-expression (in situ hybridization) in colonic MM (bracket). d, Intestinal crypts cultured over manually isolated colonic MM sheet without growth factors and imaged on day 4 (collage of micrographs, scale bar $200 \mu \mathrm{m}$ ). Magnified areas to the right show flourishing organoids (arrowhead) close to the MM membrane (black box, scale bar $100 \mu \mathrm{m}$ ) and inviable crypts farther away (red box). Images are from one of 3 biological replicates. e, Rspo2 (in situ hybridization, green) and LAMININ (immunostaining, red) in small intestine trophocytes (arrow). Bottom image is a greyscale version of the top without LAM signal and dashed outlines demarcate a single crypt. 
f, Crypts cultured with as little as $1 \mathrm{ng} / \mathrm{ml} \mathrm{rRSPO} 2$ form organoids, compared to the higher requirements for $\mathrm{rRSPO}$. Graph depicts organoid-forming efficiency with respect to $100 \mathrm{ng} / \mathrm{ml}$ of $\mathrm{rRSPO} 3$ ( $\mathrm{n}=3$ biological replicates) at 5 days. Statistical comparisons used one-way ANOVA followed by Tukey's posttest at ${ }^{* * *} \mathrm{P}<0.0001$.

\section{Figure 6: Intestinal SM, including MM, prevents BMP-driven crypt attrition.}

a, Myh11 ${ }^{\text {Cre(ER-T2) }}$,Rosa26 ${ }^{\text {DTA }}$ mice injected with TAM at P14 show reduced MM (bracket) at P21 by ACTA2 immunostaining (dotted white line=inner MP, $n=4)$. Crosses with Pdgfra ${ }^{\text {H2BeGFP }}$ show retention of Pdgfra ${ }^{\text {hi }}$ and Pdgfralo cells ( $n=3$, arrowhead points to sub-cryptal GFPlo trophocyte). Crypt epithelial proliferation (assessed $1 \mathrm{~h}$ after BrdU injection) and Olfm4-expressing ISCs were unperturbed $(n=3)$. All scale bars $(a, b, c) 50 \mu \mathrm{m}$.

b, F-actin (phalloidin)- stained epithelial whole-mounts show fewer bifid crypts (arrowheads) in Myh11 ${ }^{\text {Cre(ER-T2); }}{ }^{\text {Rosa26 }} 6^{\text {DTA }}$ pups injected with TAM at P14 and examined at P16. Data from 3 biological replicates are shown (>100 crypts counted/sample, ${ }^{*} p<0.05$ using unpaired student's t-test).

c, Immunostains show increased pSMAD1/5 in Myh11 ${ }^{\text {Cre(ER-T2) }}$;Rosa26 ${ }^{D T+}$ crypts (arrowheads), which lack pSMAD1/5 in controls (see also Fig. 1a).

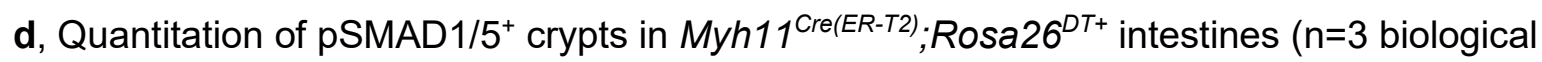
replicates, $>80$ crypts per sample, ${ }^{* *} p<0.01$ using unpaired student's t-test).

e, Model depicting the postnatal ISC niche, with ISEMFs (green) aggregating at crypt tops and MM (blue) positioning beneath the crypt base and above trophocytes (red) and MP muscle layers (Nog+=purple; Pcp4+=yellow; Osr1+=pink). The reduced BMPi reserve after SM ablation increases crypt pSMAD1/5 activity and impairs BMPi-dependent crypt fission. 


\section{SUPPLEMENTAL DATA FIGURE LEGENDS}

\section{Supplemental Data Figure 1: pSMAD and ISEMF distribution during pre- and post-natal development and phenotypes of organoid co-cultures.}

a, pSMAD1/5 concentration at the tips of nascent intestinal villi (arrowheads) and along the lengths of formed villi (red dashed outline) at E16.5 and P1 mice. Bottom image is a greyscale version of the top, with DAPI signals excluded.

b, Representative staining with the indicated antibodies in Pdgfra ${ }^{\text {H2BeGFP }}$ fetuses at E14.5 and E16.5. GFPhi ISEMFs concentrate at emerging villus tips, while GFPlo cells appear throughout the stroma. Bottom row represents same images as the top, without DAPI signals. Dotted white outlines in a and b represent inter-villus epithelium. Scale bars (a-b) $50 \mu \mathrm{m}$.

c, Passaged organoids co-cultured with ISEMFs (left, Pdgfra ${ }^{H 2 B e G F P}$ fluorescence; right, GFPhi signals in greyscale) show one of the 4 phenotypes quantified in Fig. 1d: spheroid, collapsing, budding or dead. Scale bar $500 \mu \mathrm{m}$.

d, Organoids cultured with ISEMFs and photographed on consecutive days, showing collapse of budding structures (arrowheads). Scale bar $100 \mu \mathrm{m}$.

\section{Supplemental Data Figure 2: Merged postnatal scRNA datasets from small intestine mesenchyme and identification of adult LPM and MM by in situ hybridization.}

a, Markers signifying distinct cell types (right) and global uniform manifold approximation and projection (UMAP) clustering of cell populations from merged postnatal scRNA-seq datasets.

b, Distribution of cells within the global UMAP plot with respect to the stage (left) or method (right, unfractionated vs. GFP+ cells) of mesenchymal cell isolation.

c, Adult Pdgfra ${ }^{\text {H2BeGFP }}$ duodenal Hhip expression revealed by in situ hybridization (left, RFP; right, greyscale) in the villus lamina propria (arrow), distinct from (deep to) GFP ${ }^{+}$ISEMFs, which abut the epithelium.

d, Average expression of Wnt, Wnt inhibitor, and BMP genes in postnatal intestinal stroma.

\section{Supplemental Data Figure 3: Differential gene expression, P1 muscularis propria (MP), and marker analysis of Pdgfra ${ }^{\text {H2BeGFP }}$ scRNA datasets.}

a, Representative whole-mount image from Etv1 ${ }^{\text {Cre(ER-T2) }}$;Rosa26-S-L-TdTom;Pdgfra ${ }^{\text {H2BeGFP }}$ mouse ileum, showing the distribution of sub-cryptal mesenchymal populations in relation to ICC projections, which label red from Etv ${ }^{\text {Cre }}$ and demarcate SM layers. Trophocytes lie between MM (yellow dashed line, unstained) and $\mathrm{Nog}^{+} \mathrm{Chdl1}{ }^{+}$cells (also unstained, but neural projections 
separate these cells from circular $P C P^{+} S M$ ). Purkinje neurons ${ }^{45}$ (GFPhi nuclei far from ISEMFs) are distinct from Etv1 ${ }^{\text {Cre }}$-labeled TdTom ${ }^{+}$ICC projections (red). White dashes outline approximate crypt epithelium. Scale bar $50 \mu \mathrm{m}$.

b, Representative images of small intestine crypts after 5 days of culture in a sub-optimal concentration of rRSPO1 and $100 \mathrm{ng} / \mathrm{mL}$ of the indicated BMP inhibitors. Scale bar $100 \mu \mathrm{m}$.

c, Average expression of the top 50 differentially expressed genes between P1/2 (aggregated) and P14, shown at those ages, P4+P5 (aggregated), and P9 in trophocytes (left) and $\mathrm{Nog}^{+} \mathrm{Chd} / 1^{+}$MP cells (right).

d, Numbers of informative cells of each type represented in the merged postnatal dataset. e-f, Expression at P1 of Noggin (e), Pcp4 and Osr1 ( $\mathrm{f}$ - in situ hybridization, all GFP or greyscale) showing their respective localization in successively deeper MP layers, each indicated by brackets and assisted by LAM Ab staining (red). Dashed outlines in e demarcate crypts and the boxed area is magnified at the right (top, color; bottom, greyscale). All scale bars $50 \mu \mathrm{m}$.

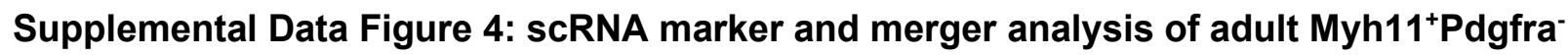 cells and adult colonic MM.}

a, Markers and graph-based UMAP clustering in $\mathrm{GFP}^{+}$cell fractions from $\mathrm{P} 14$, including average BMPi and Rspo gene expression at P14.

b, scRNA marker analysis of 2,224 adult small intestine $M y h 11^{+}$mesenchymal cells and graphbased UMAP clustering of cell populations. Hhip (red) expression principally marks LPM and Grem2 (blue) marks the substantially smaller number of recoverable MM cells.

c, The above data (b) were combined with scRNA-seq data from unfractionated adult small intestine mesenchyme ${ }^{33}$ (GEO series GSE130681), where molecular markers identified each cell type in the integrated dataset. Number of cells per identified cell type and average Bmpi and Rspo gene expression shown at right.

\section{Supplemental Data Figure 5: Investigation of MM functions and human RSPO potency.}

a, small intestine crypts cultured over manually stripped colonic MM develop into spheroidal structures within 1 day $(n=3)$. The image is a collage assembled from multiple microscopic fields (scale bar $200 \mu \mathrm{m}$ ). Inset magnifies one such field within the Matrigel drop (scale bar $100 \mu \mathrm{m}$ ). b, Rspo1 mRNA (in situ hybridization, red) in adult duodenal serosa (bracket). Scale bar $50 \mu \mathrm{m}$. c, Murine small intestinal crypts were cultured in media containing murine rEGF, rNOG, and different concentrations of the indicated human RSPOs ( $n=3$ biological replicates). Organoid 
bioRxiv preprint doi: https://doi.org/10.1101/2021.09.28.462142; this version posted September 30, 2021. The copyright holder for this preprint (which was not certified by peer review) is the author/funder, who has granted bioRxiv a license to display the preprint in perpetuity. It is made available under aCC-BY-ND 4.0 International license.

McCarthy et al, 2021

formation is represented with respect to the number of organoids formed in $100 \mathrm{ng} / \mathrm{ml} \mathrm{hRSPO}$. Statistical significance was determined using one-way ANOVA followed by Tukey's posttest at ${ }^{* * *} \mathrm{P}<0.001$ and ${ }^{*} \mathrm{P}<0.05$.

d, F-actin (phalloidin)- stained epithelial whole-mounts show fewer bifid crypts (arrowheads) in Myh11 ${ }^{\text {Cre(ER-T2) }}$;Rosa26 $6^{\text {DTA }}$ pups injected with TAM at P14 and examined at P21. Data from 1 animal.

e, Reduced small intestine (SI) length at P16 in Myh11 ${ }^{\text {Cre(ER-T2);Rosa26 }}{ }^{\text {DTA }}$ pups treated with tamoxifen at P14. Unpaired students t-test, ${ }^{* *} \mathrm{p}<0.01, \mathrm{n}=3\left(\mathrm{Myh11}^{\mathrm{Cre}}+\right)$ or $4\left(\right.$ Rosa26 $^{\text {DTA }}$ only, no Cre). 

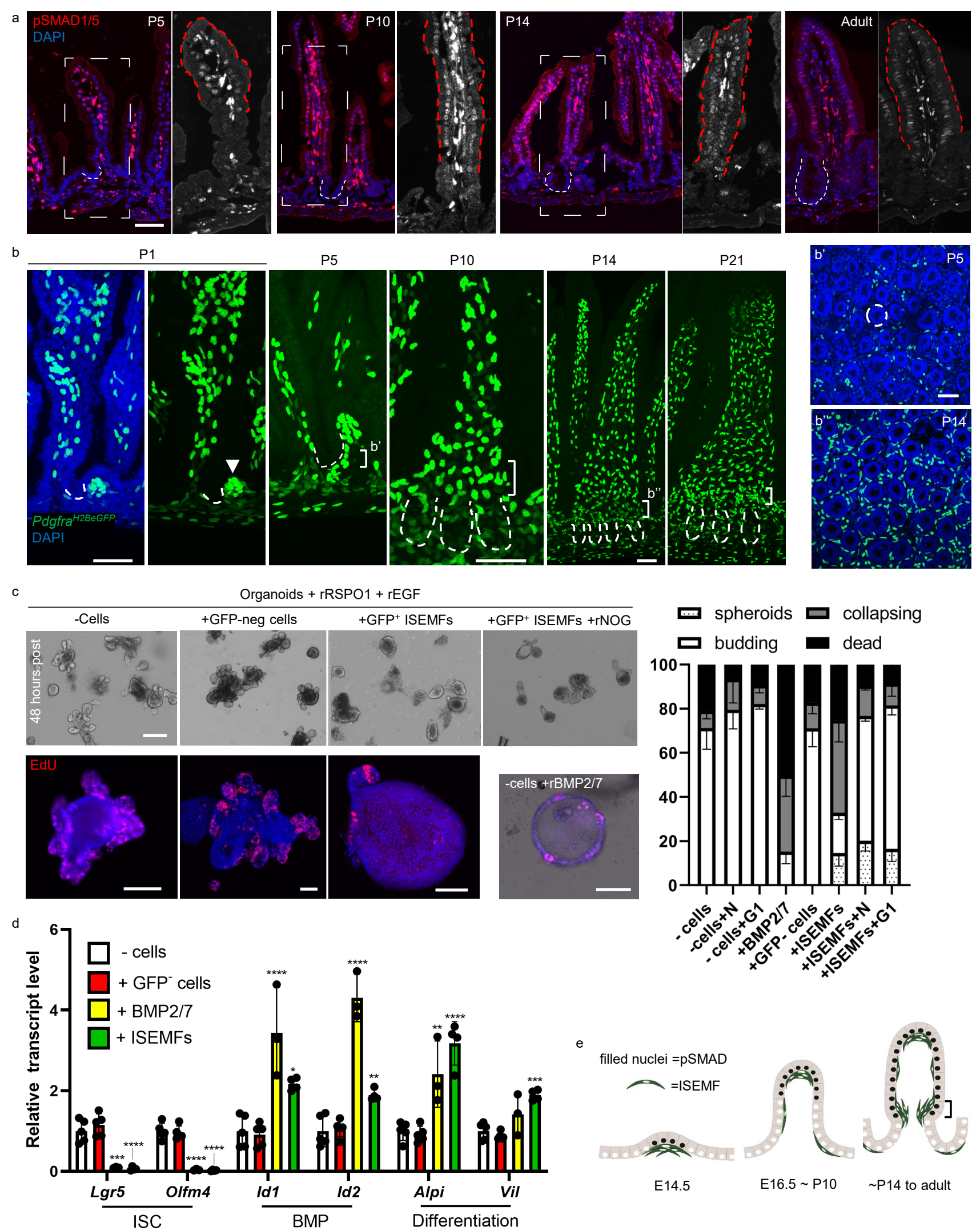

filled nuclei $=p S M A D$

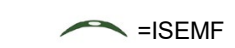

Figure 1: Postnatal epithelial BMP signaling coincides with isthmus ISEMF condensation. 


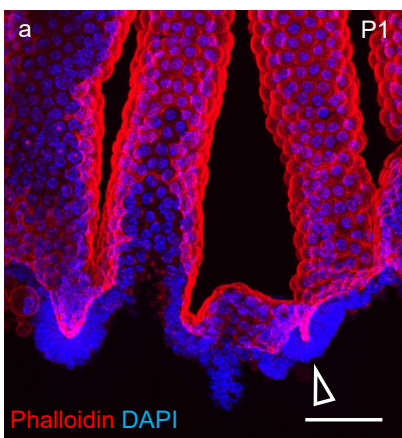

b

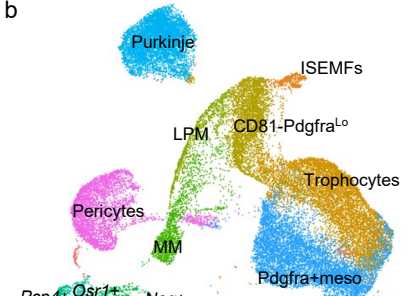

Pcp4+ $\mathrm{Osr}_{\mathrm{N} 1+}+\mathrm{Nog}+$

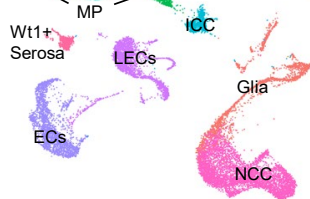

NCC

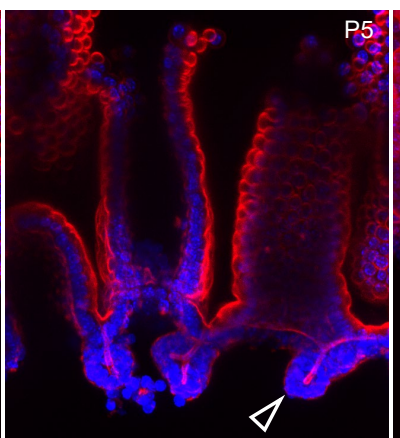

C

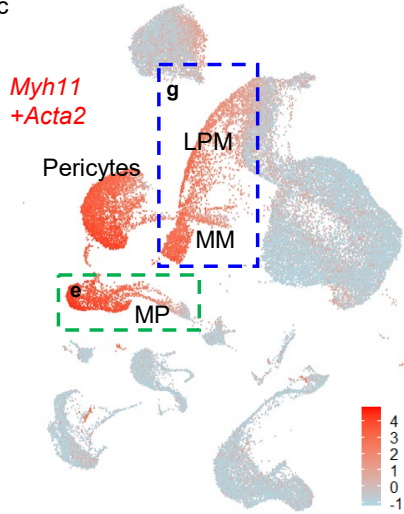

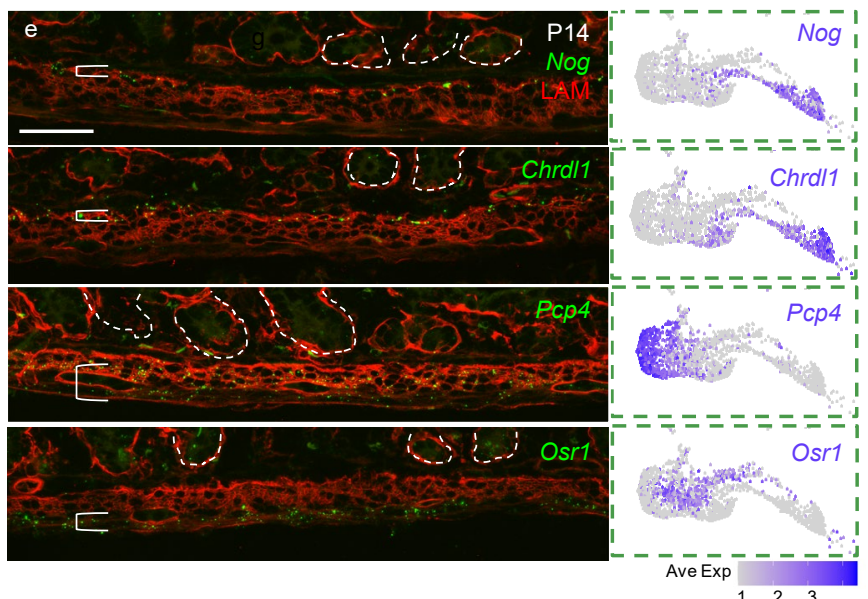
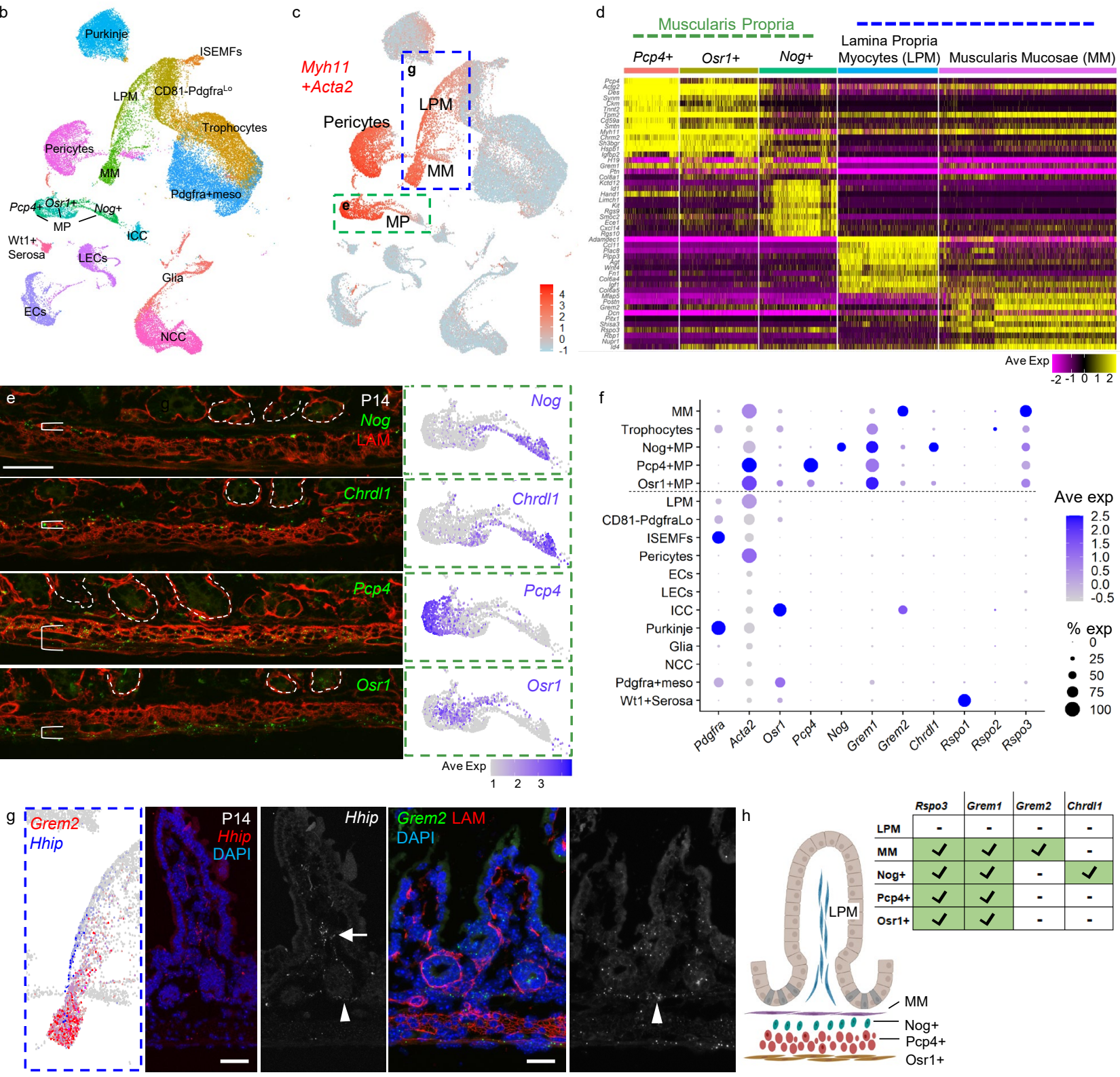

Figure 2: Diverse smooth muscle (SM) populations in postnatal intestinal mesenchyme. 

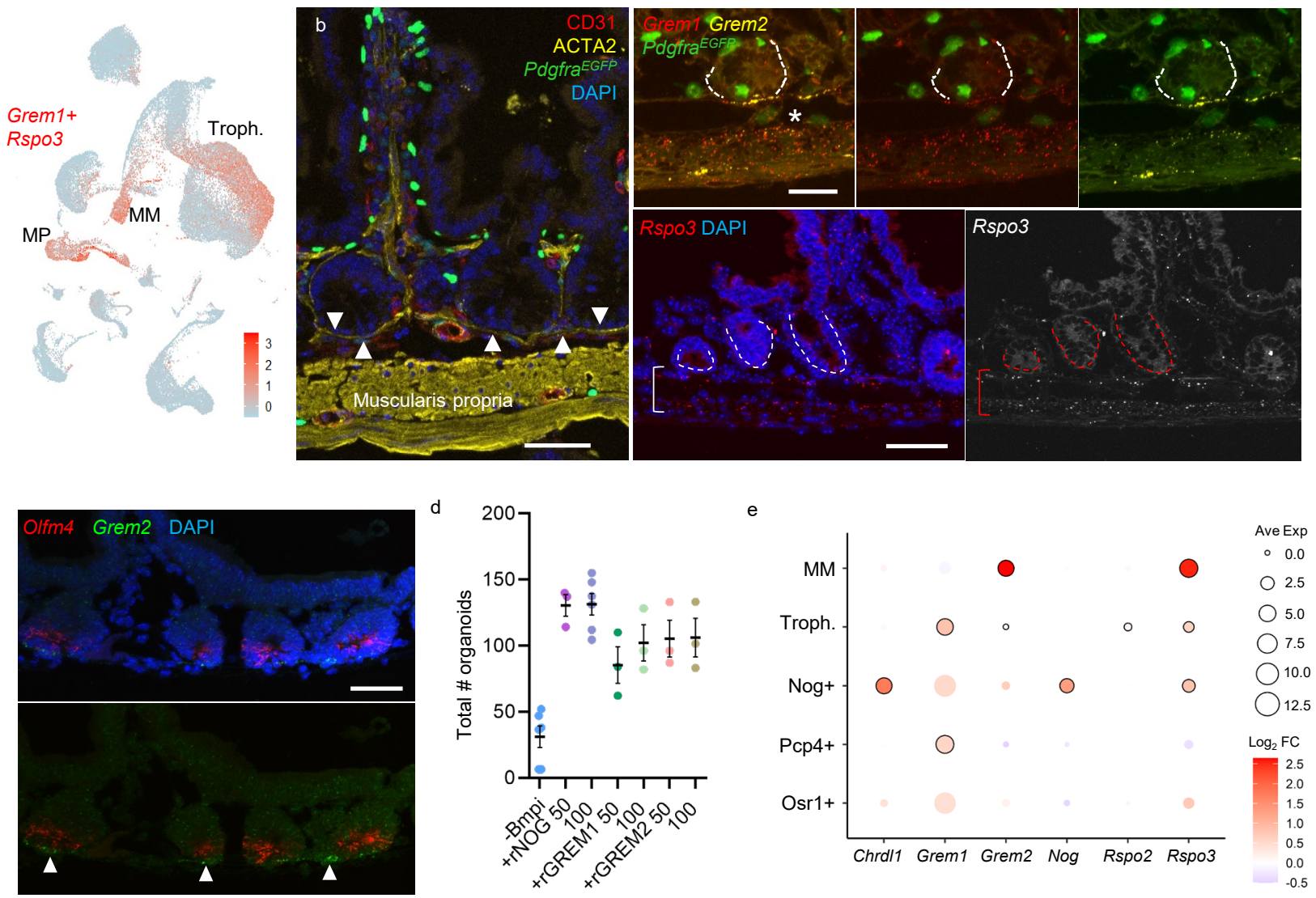

Figure 3: Sub-cryptal mesenchymal cells together constitute an ISC signaling center. 

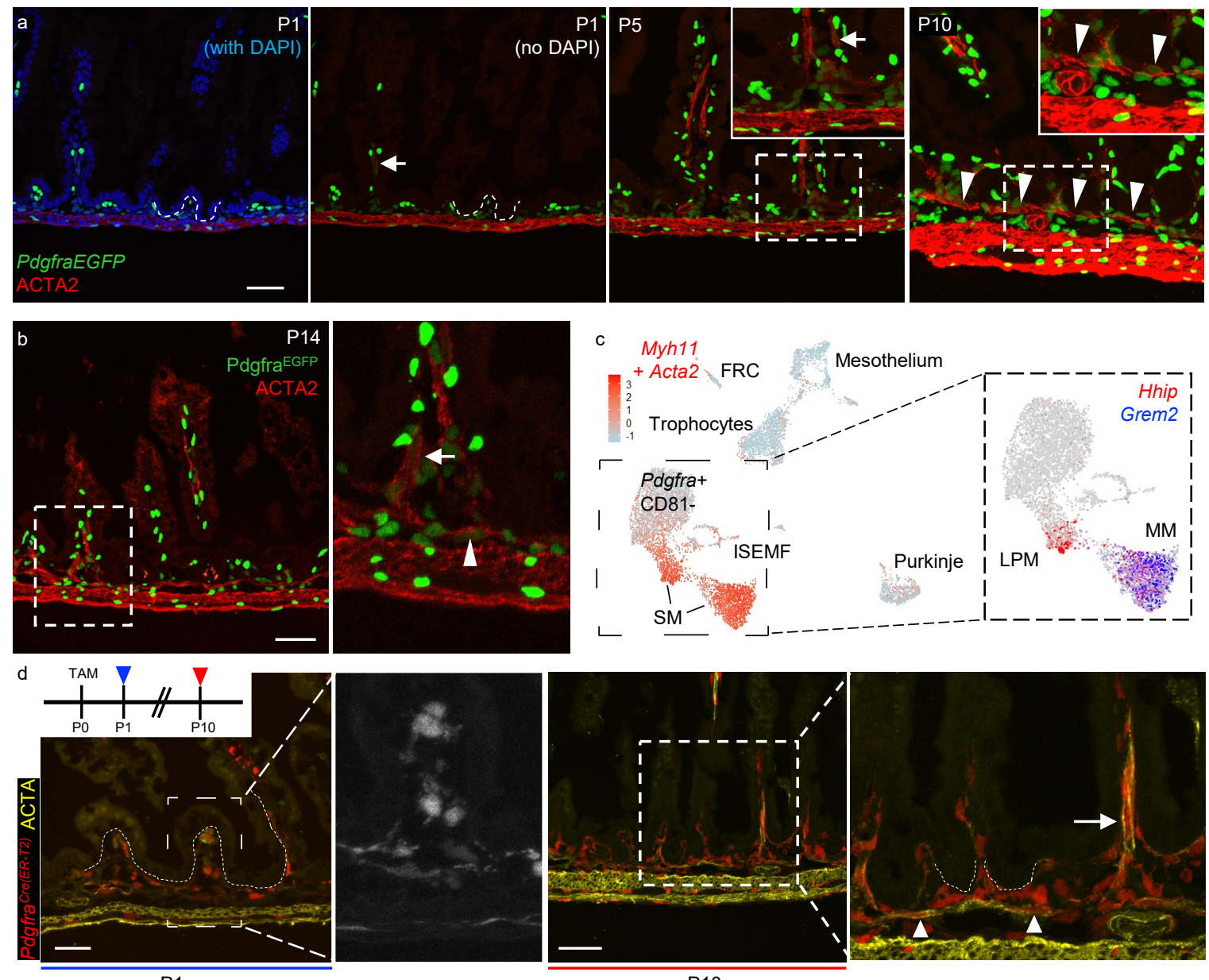

P10

Figure 4: Muscularis mucosae (MM) arises postnatally from native Pdgfra+ precursors. 

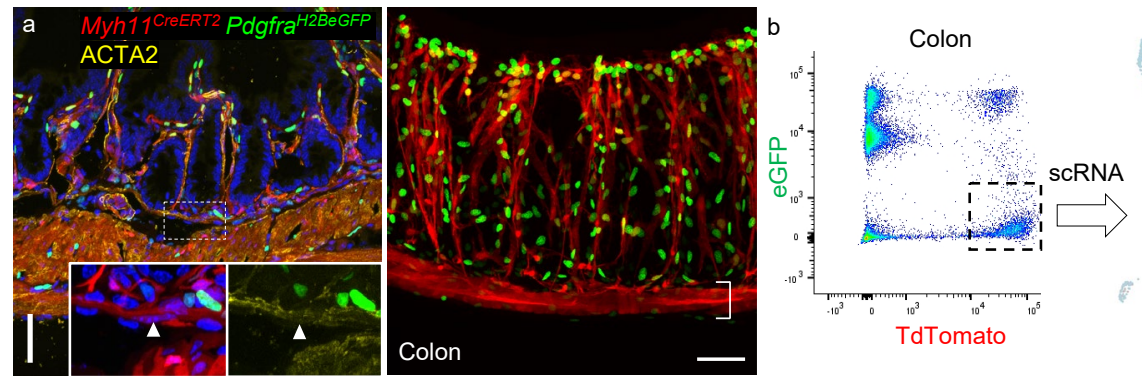

ECs

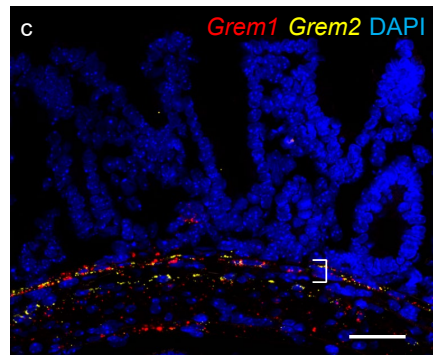

d
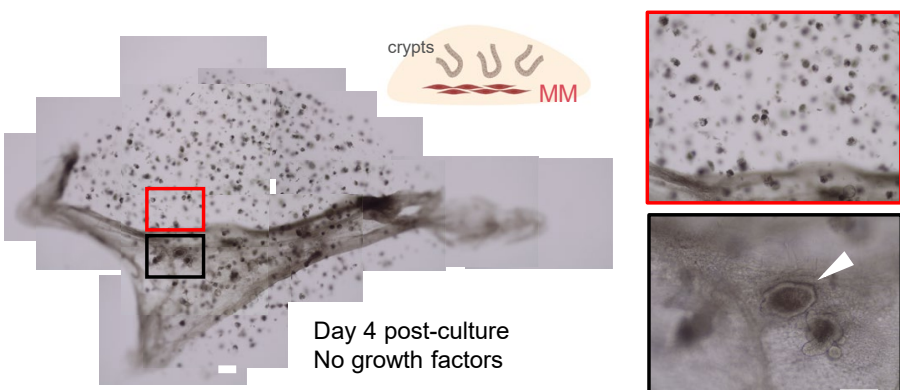

Grem1

+ Grem2

+ Rspo3
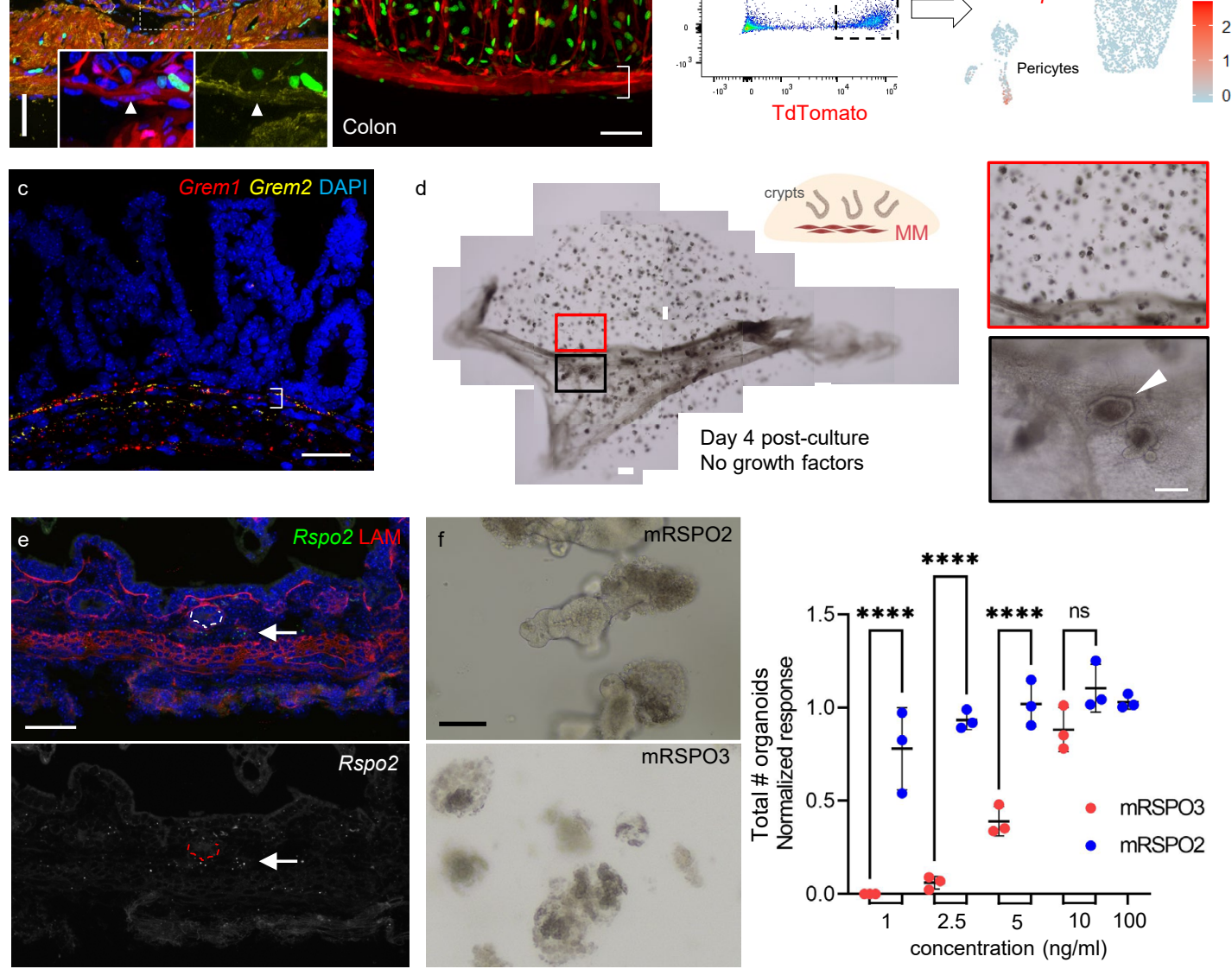

Figure 5: Adult MM and trophocytes together support organoid growth in vitro. 

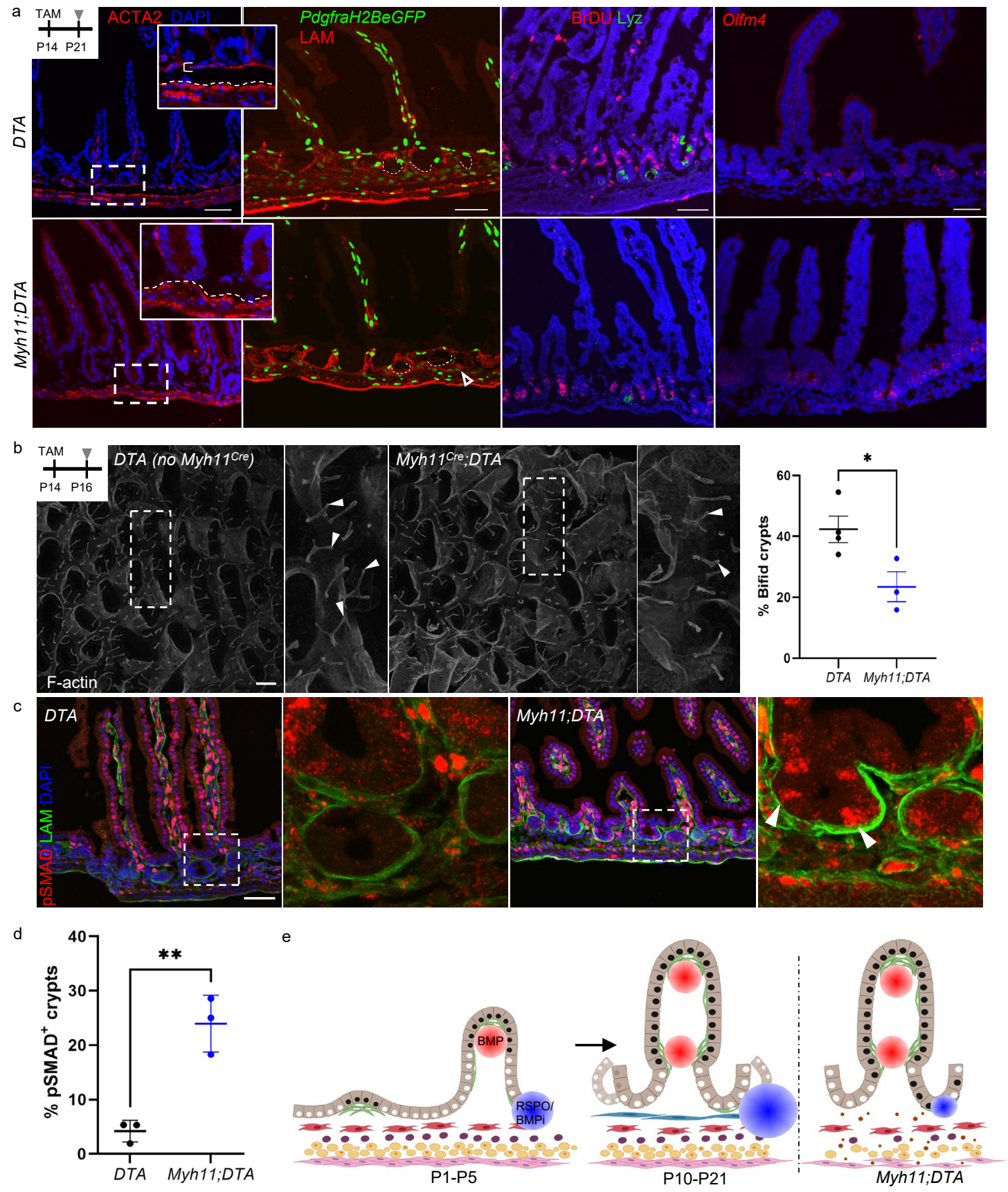

Figure 6: Intestinal SM, including MM, prevents BMP-driven crypt attrition. 